\title{
Mueller-Navelet small-cone jets at LHC in next-to-leading BFKL
}

\author{
F. Caporale ${ }^{1 \dagger}$, D.Yu. Ivanov ${ }^{2 \uparrow}$, B. Murdaca ${ }^{1 \dagger}$ and A. Papa ${ }^{1 \dagger}$ \\ ${ }^{1}$ Dipartimento di Fisica, Università della Calabria, \\ and Istituto Nazionale di Fisica Nucleare, Gruppo collegato di Cosenza, \\ I-87036 Arcavacata di Rende, Cosenza, Italy \\ 2 Sobolev Institute of Mathematics and Novosibirsk State University, \\ 630090 Novosibirsk, Russia
}

\begin{abstract}
We consider within QCD collinear factorization the process $p+p \rightarrow$ jet + jet $+X$, where two forward high $-p_{T}$ jets are produced with a large separation in rapidity $\Delta y$ (Mueller-Navelet jets). In this case the (calculable) hard part of the reaction receives large higher-order corrections $\sim \alpha_{s}^{n}(\Delta y)^{n}$, which can be accounted for in the BFKL approach with next-to-leading logarithmic accuracy, including contributions $\sim \alpha_{s}^{n}(\Delta y)^{n-1}$. We calculate several observables related with this process, using the next-to-leading order jet vertices, recently calculated in the approximation of small aperture of the jet cone in the pseudorapidity-azimuthal angle plane.
\end{abstract}

${ }^{\dagger}$ e-mail address: francesco.caporale, beatrice.murdaca, alessandro.papa @fis.unical.it

^e-mail address: d-ivanov@math.nsc.ru 


\section{Introduction}

We consider the inclusive production at high energies of two forward high- $k_{T}$ jets in protonproton collisions,

$$
p\left(p_{1}\right)+p\left(p_{2}\right) \rightarrow \operatorname{jet}\left(k_{J_{1}}\right)+\operatorname{jet}\left(k_{J_{2}}\right)+X,
$$

which are detected in the fragmentation regions of two colliding protons, $p\left(p_{1}\right)$ and $p\left(p_{2}\right)$, and are separated by a large interval of rapidity $\Delta y$, the so-called Mueller-Navelet (MN) process [1]. This is considered as an important process for the manifestation of the BFKL [2] dynamics at hadron colliders, such as Tevatron and LHC.

The theoretical description of this process is based on the QCD collinear factorization. Neglecting higher-twist contributions (terms suppressed with respect to the leading scaling asymptotic by additional inverse powers of the hard scale), the process can be viewed as started by two hadrons each emitting one parton, according to its parton distribution function (PDF), with the subsequent partonic hard scattering, see Fig. 1. Collinear factorization allows to systematically resum the logarithms of the hard scale, calculating the standard DGLAP evolution [3] of the PDFs and the fixed-order radiative corrections to the parton scattering cross section.

On the other side, in our kinematics at large squared center-of-mass energy $\sqrt{s}$, when the rapidity gap between the two produced forward jets is large, the BFKL resummation of energy logarithms comes into play, since large logarithms of the energy compensate the small QCD coupling and must be resummed to all orders of perturbation theory.

In comparison to the fixed-order DGLAP calculation, where an almost back-to-back emission is expected, the BFKL calculation assumes more emission of partons between the two jets and leads generically to a larger cross-section and to a reduced azimuthal correlation between the detected two forward jets.

At present the BFKL approach provides a general framework for the resummation of energy logarithms in the leading logarithmic approximation (LLA), which means resummation of all terms $\left(\alpha_{s} \ln (s)\right)^{n}$, and in the next-to-leading logarithmic approximation (NLA), which means resummation of all terms $\alpha_{s}\left(\alpha_{s} \ln (s)\right)^{n}$. Such resummation is process-independent and is encoded in the Green's function for the interaction of two Reggeized gluons. The Green's function is determined through the BFKL equation, which is an iterative integral equation, whose kernel is known at the next-to-leading order (NLO) both for forward scattering (i.e. for $t=0$ and color singlet in the $t$-channel) [4, 5] and for any fixed (not growing with energy) momentum transfer $t$ and any possible two-gluon color state in the $t$-channel [6, 7, 8].

The process-dependent part of the information needed for constructing the cross section for the production of Mueller-Navelet jets is contained in the impact factors for the transition from the colliding parton to the forward jet (the so called "jet vertex"). Mueller-Navelet jet vertices were calculated with NLO accuracy in [9]. The results of [9] were then used in [10, 11] for a numerical estimation in the NLA of the cross section for Mueller-Navelet jets at LHC and for the analysis of the azimuthal correlation of the produced jets. This numerical analysis followed previous ones [12, 13] based on the inclusion of NLO effects only in the Green's 
functions. Recently we performed a new calculation [14] of the jet impact factor, confirming the results of [9].

Although NLO jet vertices were obtained for a general jet algorithm, the implementation of these results into the cross section and the other Mueller-Navelet jet observables calculation requires a rather complicated numerical evaluation, see [10]. Recently the NLO impact factor for the production of forward jets was calculated by two of us [15] in the "small-cone" approximation (SCA) [16, 17], i.e. for small jet cone aperture in the rapidity-azimuthal angle plane. The use of the SCA allowed to get a simple analytic result for the jet vertices in the so-called $(\nu, n)$-representation (the jet vertices projected on the eigenfunctions of the BFKL kernel) which can be easily implemented in numerical calculations. It is the aim of the present paper to obtain predictions for the Mueller-Navelet jet process cross section and for the azimuthal angle decorrelation observables in the SCA using the results of [15].

Before we proceed, let us further comment on the theoretical input behind our approach. Our basic assumption is to neglect entirely all contributions (higher twists) that are suppressed by additional inverse powers of the jet transverse momenta. Due to this, we work in collinear factorization and express our results in terms of the usual collinear PDFs and the hard part, i.e. the cross section for the inclusive production of two jets, initiated by two partons collinear to the incident protons.

The hard part is calculated within the BFKL NLA approach, which means that we neglect contributions that are suppressed by additional inverse powers of the energy and resum to all orders in a model-independent way the leading and the first subleading energy logarithms.

Another approach, widely used in small- $x$ phenomenology, is based on the high-energy, or $k_{T}$-factorization [18]. In this case cross sections are expressed in terms of the unintegrated gluon density, which depends on both the longitudinal $x$ and the transverse $k_{T}$-part of the gluon momentum. The $k_{T}$-factorization method allows also to resum the leading (and potentially first nonleading) logarithms of the energy, and what is more important, it allows to take into account some higher-twist contributions, which could play a significant role in the description of small- $x$ processes.

In the Mueller-Navelet kinematics, each of the two jets is produced in the fragmentation region of one of the two incident protons, which ensures that the longitudinal momentum fractions of both partons that initiate the hard scattering are not small.

In other words, despite its diffractive nature, the Mueller-Navelet process does not belong to the class of small- $x$ reactions. Therefore the collinear factorization seems to be an adequate tool here. Another interesting possibility is to study processes where there is only one forward jet associated with some hard final state $X$ produced at central rapidities (say, $X$ includes one hard central jet). Such processes are initiated by small- $x$ gluons, therefore it is natural to describe them, contrary to the Mueller-Navelet process, using the $k_{T}$-factorization method, see for instance [19].

The paper is organized as follows: in the next Section we discuss the kinematics and recall the formulae for the Mueller-Navelet jet process cross section; in Section 3 we present our results; Section 4 contains the discussion of results and our conclusions. 


\section{Mueller-Navelet jet cross section}

It is convenient to define the Sudakov decomposition for the momenta of the jets; one has

$$
\begin{array}{ll}
k_{J_{1}}=x_{J_{1}} p_{1}+\frac{\vec{k}_{J_{1}}^{2}}{x_{J_{1}} s} p_{2}+k_{J_{1} \perp}, & k_{J_{1} \perp}^{2}=-\vec{k}_{J_{1}}^{2}, \\
k_{J_{2}}=x_{J_{2}} p_{2}+\frac{\vec{k}_{J_{2}}^{2}}{x_{J_{2}} s} p_{1}+k_{J_{2} \perp}, & k_{J_{2} \perp}^{2}=-\vec{k}_{J_{2}}^{2} .
\end{array}
$$

Here as power-suppressed correction we neglect the proton mass, $p_{1}^{2}=p_{2}^{2}=0$, therefore $p_{1}$ and $p_{2}$ are taken as Sudakov vectors satisfying $2\left(p_{1} p_{2}\right)=s$.

For the forward jets the longitudinal fractions $x_{J_{1,2}} \sim \mathcal{O}(1)$ are related to the jet rapidities in the center-of-mass system by

$$
\begin{array}{cl}
y_{1}=\frac{1}{2} \ln \frac{x_{J_{1}}^{2} s}{\vec{k}_{J_{1}}^{2}}, & d y_{1}=\frac{d x_{J_{1}}}{x_{J_{1}}}, \\
y_{2}=-\frac{1}{2} \ln \frac{x_{J_{2}}^{2} s}{\vec{k}_{J_{2}}^{2}}, & d y_{2}=-\frac{d x_{J_{2}}}{x_{J_{2}}},
\end{array}
$$

so that the rapidity gap between the two jets is given by

$$
\Delta y \equiv Y=\ln \frac{x_{J_{1}} x_{J_{2}} s}{\left|\vec{k}_{J_{1}}\right|\left|\vec{k}_{J_{2}}\right|} .
$$

For the Mueller-Navelet jet process, the rapidity gap $Y$ is to be taken much larger than unity, thus implying the kinematics

$$
s \gg \vec{k}_{J_{1,2}}^{2} \gg \Lambda_{Q C D}^{2},
$$

and the transverse momenta of the jets are assumed to be of similar order of magnitude, $\vec{k}_{J_{1}}^{2} \sim \vec{k}_{J_{2}}^{2}$

In QCD collinear factorization the cross section of the process (11) reads

$$
\frac{d \sigma}{d x_{J_{1}} d x_{J_{2}} d^{2} k_{J_{1}} d^{2} k_{J_{2}}}=\sum_{i, j=q, \bar{q}, g} \int_{0}^{1} d x_{1} \int_{0}^{1} d x_{2} f_{i}\left(x_{1}, \mu_{F}\right) f_{j}\left(x_{2}, \mu_{F}\right) \frac{d \hat{\sigma}_{i, j}\left(x_{1} x_{2} s, \mu_{F}\right)}{d x_{J_{1}} d x_{J_{2}} d^{2} k_{J_{1}} d^{2} k_{J_{2}}}
$$

where the $i, j$ indices specify the parton types (quarks $q=u, d, s, c, b$; antiquarks $\bar{q}=$ $\bar{u}, \bar{d}, \bar{s}, \bar{c}, \bar{b}$; or gluon $g), f_{i}\left(x, \mu_{F}\right)$ denotes the initial proton PDFs, the longitudinal fractions of the partons involved in the hard subprocess are $x_{1,2}$, as shown in Fig. 1, $\mu_{F}$ is the factorization scale, $d \hat{\sigma}_{i, j}\left(x_{1} x_{2} s, \mu_{F}\right)$ is the partonic cross section for the production of jets and $\hat{s}=x_{1} x_{2} s$ is the squared center-of-mass energy of the parton-parton collision subprocess. We use the $\overline{\mathrm{MS}}$ scheme for the ultraviolet and collinear factorizations.

At lowest order each jet is generated by a single parton having high transverse momentum and the partonic subprocess is given by an elementary two-to-two scattering. In the discussed 
Mueller-Navelet kinematics the higher-order contributions to the partonic cross section have to be resummed using BFKL approach. In the BFKL approach [2], the cross section of the hard subprocess reads

$$
\begin{aligned}
\frac{d \hat{\sigma}_{i, j}\left(x_{1} x_{2} s\right)}{d x_{J_{1}} d x_{J_{2}} d^{2} k_{J_{1}} d^{2} k_{J_{2}}}=\frac{1}{(2 \pi)^{2}} & \int \frac{d^{2} q_{1}}{\vec{q}_{1}^{2}} V_{i}\left(\vec{q}_{1}, s_{0}, x_{1} ; \vec{k}_{J_{1}}, x_{J_{1}}\right) \int \frac{d^{2} q_{2}}{\vec{q}_{2}^{2}} V_{j}\left(-\vec{q}_{2}, s_{0}, x_{2} ; \vec{k}_{J_{2}}, x_{J_{2}}\right) \\
& \times \int_{\delta-i \infty}^{\delta+i \infty} \frac{d \omega}{2 \pi i}\left(\frac{x_{1} x_{2} s}{s_{0}}\right)^{\omega} G_{\omega}\left(\vec{q}_{1}, \vec{q}_{2}\right)
\end{aligned}
$$

This representation for the cross section is valid with NLA accuracy. Here $V_{i}\left(\vec{q}_{1}, s_{0}, x_{1} ; \vec{k}_{J_{1}}, x_{J_{1}}\right)$ and $V_{j}\left(-\vec{q}_{2}, s_{0}, x_{2} ; \vec{k}_{J_{2}}, x_{J_{2}}\right)$ are the jet vertices (impact factors) describing the transitions parton $i\left(x_{1} p_{1}\right) \rightarrow$ jet $\left(k_{J_{1}}\right)$ and parton $j\left(x_{2} p_{2}\right) \rightarrow$ jet $\left(k_{J_{2}}\right)$, in the scattering off a Reggeized gluon with transverse momentum $\overrightarrow{q_{1}}$ and $\overrightarrow{q_{2}}$, respectively. The artificial scale $s_{0}$ is introduced in the BFKL approach to perform the Mellin transform from the $s$-space to the complex angular momentum plane and cancels in the full expression for the cross section with the NLA accuracy. The Green's function in (7) obeys the BFKL equation

$$
\delta^{2}\left(\vec{q}_{1}-\vec{q}_{2}\right)=\omega G_{\omega}\left(\vec{q}_{1}, \vec{q}_{2}\right)-\int d^{2} q K\left(\vec{q}_{1}, \vec{q}\right) G_{\omega}\left(\vec{q}, \vec{q}_{2}\right)
$$

where $K\left(\vec{q}_{1}, \vec{q}_{2}\right)$ is the BFKL kernel.

In what follows we proceed along the lines similar to ones used in Ref. [20]. It is convenient to work in the transverse momentum representation, defined by

$$
\begin{gathered}
\hat{\vec{q}}\left|\vec{q}_{i}\right\rangle=\vec{q}_{i}\left|\vec{q}_{i}\right\rangle, \\
\langle A \mid B\rangle=\langle A \mid \vec{k}\rangle\langle\vec{k} \mid B\rangle=\int d^{2} k A(\vec{k}) B(\vec{k}) ;
\end{gathered}
$$

the kernel of the operator $\hat{K}$ is

$$
K\left(\vec{q}_{2}, \vec{q}_{1}\right)=\left\langle\vec{q}_{2}|\hat{K}| \vec{q}_{1}\right\rangle
$$

and the equation for the Green's function reads

$$
\hat{1}=(\omega-\hat{K}) \hat{G}_{\omega},
$$

its solution being

$$
\hat{G}_{\omega}=(\omega-\hat{K})^{-1}
$$

The kernel is given as an expansion in the strong coupling,

$$
\hat{K}=\bar{\alpha}_{s} \hat{K}^{0}+\bar{\alpha}_{s}^{2} \hat{K}^{1},
$$

where

$$
\bar{\alpha}_{s}=\frac{\alpha_{s} N_{c}}{\pi}
$$

and $N_{c}$ is the number of colors. In Eq. (14) $\hat{K}^{0}$ is the BFKL kernel in the LLA, $\hat{K}^{1}$ represents the NLA correction. 
To determine the partonic cross section with NLA accuracy we need an approximate solution of Eq. (13). With the required accuracy this solution is

$$
\hat{G}_{\omega}=\left(\omega-\bar{\alpha}_{s} \hat{K}^{0}\right)^{-1}+\left(\omega-\bar{\alpha}_{s} \hat{K}^{0}\right)^{-1}\left(\bar{\alpha}_{s}^{2} \hat{K}^{1}\right)\left(\omega-\bar{\alpha}_{s} \hat{K}^{0}\right)^{-1}+\mathcal{O}\left[\left(\bar{\alpha}_{s}^{2} \hat{K}^{1}\right)^{2}\right] .
$$

The basis of eigenfunctions of the LLA kernel,

$$
\hat{K}^{0}|n, \nu\rangle=\chi(n, \nu)|n, \nu\rangle, \quad \chi(n, \nu)=2 \psi(1)-\psi\left(\frac{n}{2}+\frac{1}{2}+i \nu\right)-\psi\left(\frac{n}{2}+\frac{1}{2}-i \nu\right)
$$

is given by the following set of functions:

$$
\langle\vec{q} \mid n, \nu\rangle=\frac{1}{\pi \sqrt{2}}\left(\vec{q}^{2}\right)^{i \nu-\frac{1}{2}} e^{i n \phi},
$$

here $\phi$ is the azimuthal angle of the vector $\vec{q}$ counted from some fixed direction in the transverse space, $\cos \phi \equiv q_{x} /|\vec{q}|$. Then, the orthonormality condition takes the form

$$
\left\langle n^{\prime}, \nu^{\prime} \mid n, \nu\right\rangle=\int \frac{d^{2} q}{2 \pi^{2}}\left(\vec{q}^{2}\right)^{i \nu-i \nu^{\prime}-1} e^{i\left(n-n^{\prime}\right) \phi}=\delta\left(\nu-\nu^{\prime}\right) \delta_{n n^{\prime}} .
$$

The action of the full NLA BFKL kernel on these functions may be expressed as follows:

$$
\begin{aligned}
\hat{K}|n, \nu\rangle & =\bar{\alpha}_{s}\left(\mu_{R}\right) \chi(n, \nu)|n, \nu\rangle+\bar{\alpha}_{s}^{2}\left(\mu_{R}\right)\left(\chi^{(1)}(n, \nu)+\frac{\beta_{0}}{4 N_{c}} \chi(n, \nu) \ln \left(\mu_{R}^{2}\right)\right)|n, \nu\rangle \\
& +\bar{\alpha}_{s}^{2}\left(\mu_{R}\right) \frac{\beta_{0}}{4 N_{c}} \chi(n, \nu)\left(i \frac{\partial}{\partial \nu}\right)|n, \nu\rangle,
\end{aligned}
$$

where $\mu_{R}$ is the renormalization scale of the QCD coupling, the first term represents the action of LLA kernel, while the second and the third ones stand for the diagonal and the non-diagonal parts of the NLA kernel and we have used

$$
\beta_{0}=\frac{11 N_{c}}{3}-\frac{2 n_{f}}{3}
$$

where $n_{f}$ is the number of active quark flavors.

The function $\chi^{(1)}(n, \nu)$, calculated in [21] (see also [22]), is conveniently represented in the form

$$
\chi^{(1)}(n, \nu)=-\frac{\beta_{0}}{8 N_{c}}\left(\chi^{2}(n, \nu)-\frac{10}{3} \chi(n, \nu)-i \chi^{\prime}(n, \nu)\right)+\bar{\chi}(n, \nu),
$$

where

$$
\begin{gathered}
\bar{\chi}(n, \nu)=-\frac{1}{4}\left[\frac{\pi^{2}-4}{3} \chi(n, \nu)-6 \zeta(3)-\chi^{\prime \prime}(n, \nu)+2 \phi(n, \nu)+2 \phi(n,-\nu)\right. \\
\left.+\frac{\pi^{2} \sinh (\pi \nu)}{2 \nu \cosh ^{2}(\pi \nu)}\left(\left(3+\left(1+\frac{n_{f}}{N_{c}^{3}}\right) \frac{11+12 \nu^{2}}{16\left(1+\nu^{2}\right)}\right) \delta_{n 0}-\left(1+\frac{n_{f}}{N_{c}^{3}}\right) \frac{1+4 \nu^{2}}{32\left(1+\nu^{2}\right)} \delta_{n 2}\right)\right],
\end{gathered}
$$




$$
\begin{gathered}
\phi(n, \nu)=-\int_{0}^{1} d x \frac{x^{-1 / 2+i \nu+n / 2}}{1+x}\left[\frac{1}{2}\left(\psi^{\prime}\left(\frac{n+1}{2}\right)-\zeta(2)\right)+\operatorname{Li}_{2}(x)+\operatorname{Li}_{2}(-x)\right. \\
\left.+\ln x\left(\psi(n+1)-\psi(1)+\ln (1+x)+\sum_{k=1}^{\infty} \frac{(-x)^{k}}{k+n}\right)+\sum_{k=1}^{\infty} \frac{x^{k}}{(k+n)^{2}}\left(1-(-1)^{k}\right)\right] \\
=\sum_{k=0}^{\infty} \frac{(-1)^{k+1}}{k+(n+1) / 2+i \nu}\left[\psi^{\prime}(k+n+1)-\psi^{\prime}(k+1)+(-1)^{k+1}\left(\beta^{\prime}(k+n+1)+\beta^{\prime}(k+1)\right)\right. \\
\left.-\frac{1}{k+(n+1) / 2+i \nu}(\psi(k+n+1)-\psi(k+1))\right], \\
\beta^{\prime}(z)=\frac{1}{4}\left[\psi^{\prime}\left(\frac{z+1}{2}\right)-\psi^{\prime}\left(\frac{z}{2}\right)\right], \quad \operatorname{Li}_{2}(x)=-\int_{0}^{x} d t \frac{\ln (1-t)}{t} .
\end{gathered}
$$

Here and below $\chi^{\prime}(n, \nu)=d \chi(n, \nu) / d \nu$ and $\chi^{\prime \prime}(n, \nu)=d^{2} \chi(n, \nu) / d^{2} \nu$.

For the quark and the gluon jet vertices in (7) the projection onto the eigenfunctions of LO BFKL kernel, i.e. the transfer to the $(\nu, n)$-representation, is done as follows:

$$
\begin{aligned}
& \frac{V\left(\vec{q}_{1}\right)}{\vec{q}_{1}^{2}}=\sum_{n=-\infty}^{+\infty} \int_{-\infty}^{+\infty} d \nu \Phi_{1}(\nu, n)\left\langle n, \nu \mid \vec{q}_{1}\right\rangle, \quad \frac{V\left(-\vec{q}_{2}\right)}{\vec{q}_{2}^{2}}=\sum_{n=-\infty}^{+\infty} \int_{-\infty}^{+\infty} d \nu \Phi_{2}(\nu, n)\left\langle\vec{q}_{2} \mid n, \nu\right\rangle \\
& \Phi_{1}(\nu, n)=\int d^{2} q_{1} \frac{V\left(\vec{q}_{1}\right)}{\vec{q}_{1}^{2}} \frac{1}{\pi \sqrt{2}}\left(\vec{q}_{1}^{2}\right)^{i \nu-\frac{1}{2}} e^{i n \phi_{1}} \\
& \Phi_{2}(\nu, n)=\int d^{2} q_{2} \frac{V\left(-\vec{q}_{2}\right)}{\vec{q}_{2}^{2}} \frac{1}{\pi \sqrt{2}}\left(\vec{q}_{2}^{2}\right)^{-i \nu-\frac{1}{2}} e^{-i n \phi_{2}}
\end{aligned}
$$

The vertices can be represented as an expansion in $\alpha_{s}$,

$$
\Phi_{1,2}(n, \nu)=\alpha_{s}\left(\mu_{R}\right) v_{1,2}(n, \nu)+\alpha_{s}^{2}\left(\mu_{R}\right) v_{1,2}^{(1)}(n, \nu) .
$$

In Eqs. (25) and (26) we suppressed for brevity the partonic indices $i, j$ and the other arguments in $v_{1,2}$. The explicit forms of LLA and NLA jet vertices in the $(\nu, n)$-representation both for the quark and gluon cases can be found in [15]. In particular for the LLA quark vertices one has

$$
\begin{aligned}
& v_{1}^{q}(n, \nu)=2 \sqrt{\frac{C_{F}}{C_{A}}}\left(\vec{k}_{J_{1}}^{2}\right)^{i \nu-3 / 2} e^{i n \phi_{J_{1}}} \delta\left(x_{J_{1}}-x_{1}\right), \\
& v_{2}^{q}(n, \nu)=2 \sqrt{\frac{C_{F}}{C_{A}}}\left(\vec{k}_{J_{2}}^{2}\right)^{-i \nu-3 / 2} e^{-i n\left(\phi_{J_{2}}+\pi\right)} \delta\left(x_{J_{2}}-x_{2}\right),
\end{aligned}
$$

where $C_{A}=N_{c}, C_{F}=\left(N_{c}^{2}-1\right) / 2 N_{c}$, the angle $\phi_{J_{2}}+\pi$ in the last equation appears due to the fact that the Reggeon momentum which enters the second vertex is $-\vec{q}_{2}$. Note that in LLA vertices the partonic and the jet longitudinal momentum fractions coincide. 
The partonic cross section can be written with NLA accuracy as follows

$$
\begin{aligned}
& \frac{d \hat{\sigma}\left(x_{1} x_{2} s\right)}{d x_{J_{1}} d x_{J_{2}} d^{2} k_{J_{1}} d^{2} k_{J_{2}}}=\frac{1}{(2 \pi)^{2}} \sum_{n=-\infty}^{+\infty} \int_{-\infty}^{+\infty} d \nu\left(\frac{x_{1} x_{2} s}{s_{0}}\right)^{\bar{\alpha}_{s}\left(\mu_{R}\right) \chi(n, \nu)} \\
& \times \alpha_{s}^{2}\left(\mu_{R}\right) v_{1}(n, \nu) v_{2}(n, \nu)\left[1+\alpha_{s}\left(\mu_{R}\right)\left(\frac{v_{1}^{(1)}(n, \nu)}{v_{1}(n, \nu)}+\frac{v_{2}^{(1)}(n, \nu)}{v_{2}(n, \nu)}\right)\right. \\
& +\bar{\alpha}_{s}^{2}\left(\mu_{R}\right) \ln \left(\frac{x_{1} x_{2} s}{s_{0}}\right)\left(\bar{\chi}(n, \nu)+\frac{\beta_{0}}{8 N_{c}} \chi(n, \nu)\left[-\chi(n, \nu)+\frac{10}{3}\right.\right. \\
& \left.\left.\left.+i \frac{d \ln \left(\frac{v_{1}(n, \nu)}{v_{2}(n, \nu)}\right)}{d \nu}+2 \ln \mu_{R}^{2}\right]\right)\right] .
\end{aligned}
$$

For the subsequent calculation it is convenient to make the substitution

$$
\left(\frac{x_{1} x_{2} s}{s_{0}}\right)^{\bar{\alpha}_{s}\left(\mu_{R}\right) \chi(n, \nu)}=\left(\frac{x_{J_{1}} x_{J_{2}} s}{s_{0}}\right)^{\bar{\alpha}_{s}\left(\mu_{R}\right) \chi(n, \nu)}\left(\frac{x_{1}}{x_{J_{1}}}\right)^{\bar{\alpha}_{s}\left(\mu_{R}\right) \chi(n, \nu)}\left(\frac{x_{2}}{x_{J_{2}}}\right)^{\bar{\alpha}_{s}\left(\mu_{R}\right) \chi(n, \nu)}
$$

and to assign the last two factors in the r.h.s. to the corresponding jet vertices. This procedure affects only the NLA parts of the jet vertices, since for the LLA vertices $x_{i}=x_{J_{i}}$. Also with NLA accuracy, one can make in (28) the replacement

$$
\ln \left(\frac{x_{1} x_{2} s}{s_{0}}\right) \rightarrow \ln \left(\frac{x_{J_{1}} x_{J_{2}} s}{s_{0}}\right) .
$$

This procedure allows to perform in the MN-jet cross section first the integration over partonic momentum fractions, before taking the sum over $n$ and the integration over $\nu$; it allows also to consider together the contributions of quarks and gluons to the jet vertices.

The differential cross section has the form

$$
\frac{d \sigma}{d y_{J_{1}} d y_{J_{2}} d\left|\vec{k}_{J_{1}}\right| d\left|\vec{k}_{J_{2}}\right| d \phi_{J_{1}} d \phi_{J_{2}}}=\frac{1}{(2 \pi)^{2}}\left[\mathcal{C}_{0}+\sum_{n=1}^{\infty} 2 \cos (n \phi) \mathcal{C}_{n}\right]
$$

where $\phi=\phi_{J_{1}}-\phi_{J_{2}}-\pi$, and

$$
\mathcal{C}_{m}=\int_{0}^{2 \pi} d \phi_{J_{1}} \int_{0}^{2 \pi} d \phi_{J_{2}} \cos \left[m\left(\phi_{J_{1}}-\phi_{J_{2}}-\pi\right)\right] \frac{d \sigma}{d y_{J_{1}} d y_{J_{2}} d\left|\vec{k}_{J_{1}}\right| d\left|\vec{k}_{J_{2}}\right| d \phi_{J_{1}} d \phi_{J_{2}}}
$$

In particular, taking into account the Jacobian of the transformation from the variables $\vec{k}_{J_{i}}, x_{J_{i}}$ to the variables $\left|\vec{k}_{J_{i}}\right|, y_{J_{i}}$, and the $\nu$-dependence of LLA jet vertices, see (27), we get

$$
\begin{aligned}
& \mathcal{C}_{n}=\frac{x_{J_{1}} x_{J_{2}}}{\left|\vec{k}_{J_{1}}\right|\left|\vec{k}_{J_{2}}\right|} \int_{-\infty}^{+\infty} d \nu\left(\frac{x_{J_{1}} x_{J_{2}} s}{s_{0}}\right)^{\bar{\alpha}_{s}\left(\mu_{R}\right) \chi(n, \nu)} \\
& \times \alpha_{s}^{2}\left(\mu_{R}\right) c_{1}\left(n, \nu,\left|\vec{k}_{J_{1}}\right|, x_{J_{1}}\right) c_{2}\left(n, \nu,\left|\vec{k}_{J_{2}}\right|, x_{J_{2}}\right)
\end{aligned}
$$




$$
\begin{gathered}
\times\left[1+\alpha_{s}\left(\mu_{R}\right)\left(\frac{c_{1}^{(1)}\left(n, \nu,\left|\vec{k}_{J_{1}}\right|, x_{J_{1}}\right)}{c_{1}\left(n, \nu,\left|\vec{k}_{J_{1}}\right|, x_{J_{1}}\right)}+\frac{c_{2}^{(1)}\left(n, \nu,\left|\vec{k}_{J_{2}}\right|, x_{J_{2}}\right)}{c_{2}\left(n, \nu,\left|\vec{k}_{J_{2}}\right|, x_{J_{2}}\right)}\right)\right. \\
\left.+\bar{\alpha}_{s}^{2}\left(\mu_{R}\right) \ln \left(\frac{x_{J_{1}} x_{J_{2}} s}{s_{0}}\right)\left(\bar{\chi}(n, \nu)+\frac{\beta_{0}}{8 C_{A}} \chi(n, \nu)\left(-\chi(n, \nu)+\frac{10}{3}+\ln \frac{\mu_{R}^{4}}{\vec{k}_{J_{1}}^{2} \vec{k}_{J_{2}}^{2}}\right)\right)\right],
\end{gathered}
$$

where

$$
\begin{aligned}
& c_{1}(n, \nu,|\vec{k}|, x)=2 \sqrt{\frac{C_{F}}{C_{A}}}\left(\vec{k}^{2}\right)^{i \nu-1 / 2}\left(\frac{C_{A}}{C_{F}} f_{g}\left(x, \mu_{F}\right)+\sum_{a=q, \bar{q}} f_{q}\left(x, \mu_{F}\right)\right), \\
& c_{2}(n, \nu,|\vec{k}|, x)=\left[c_{1}(n, \nu,|\vec{k}|, x)\right]^{*} \\
& c_{1}^{(1)}(n, \nu,|\vec{k}|, x)=\frac{1}{\pi} \sqrt{\frac{C_{F}}{C_{A}}}\left(\vec{k}^{2}\right)^{i \nu-1 / 2} \int_{x}^{1} \frac{d \zeta}{\zeta} \zeta^{-\bar{\alpha}_{s}\left(\mu_{R}\right) \chi(n, \nu)}\left\{\sum_{a=q, \bar{q}} f_{a}\left(\frac{x}{\zeta}\right)\right. \\
& \times\left[\left(P_{q q}(\zeta)+\frac{C_{A}}{C_{F}} P_{g q}(\zeta)\right) \ln \frac{\vec{k}^{2}}{\mu_{F}^{2}}-2 \zeta^{-2 \gamma} \ln R\left\{P_{q q}(\zeta)+P_{g q}(\zeta)\right\}-\frac{\beta_{0}}{2} \ln \frac{\vec{k}^{2}}{\mu_{R}^{2}} \delta(1-\zeta)\right. \\
& +C_{A} \delta(1-\zeta)\left(\chi(n, \gamma) \ln \frac{s_{0}}{\vec{k}^{2}}+\frac{85}{18}+\frac{\pi^{2}}{2}+\frac{1}{2}\left(\psi^{\prime}\left(1+\gamma+\frac{n}{2}\right)-\psi^{\prime}\left(\frac{n}{2}-\gamma\right)-\chi^{2}(n, \gamma)\right)\right) \\
& +\left(1+\zeta^{2}\right)\left\{C_{A}\left(\frac{\left(1+\zeta^{-2 \gamma}\right) \chi(n, \gamma)}{2(1-\zeta)_{+}}-\zeta^{-2 \gamma}\left(\frac{\ln (1-\zeta)}{1-\zeta}\right)_{+}\right)+\left(C_{F}-\frac{C_{A}}{2}\right)\left[\frac{\bar{\zeta}}{\zeta^{2}} I_{2}-\frac{2 \ln \zeta}{1-\zeta}\right.\right. \\
& \left.\left.+2\left(\frac{\ln (1-\zeta)}{1-\zeta}\right)_{+}\right]\right\}+\delta(1-\zeta)\left(C_{F}\left(3 \ln 2-\frac{\pi^{2}}{3}-\frac{9}{2}\right)-\frac{5 n_{f}}{9}\right) \\
& \left.+C_{A} \zeta+C_{F} \bar{\zeta}+\frac{1+\bar{\zeta}^{2}}{\zeta}\left(C_{A} \frac{\bar{\zeta}}{\zeta} I_{1}+2 C_{A} \ln \frac{\bar{\zeta}}{\zeta}+C_{F} \zeta^{-2 \gamma}(\chi(n, \gamma)-2 \ln \bar{\zeta})\right)\right] \\
& +f_{g}\left(\frac{x}{\zeta}\right) \frac{C_{A}}{C_{F}} \\
& \times\left[\left(P_{g g}(\zeta)+2 n_{f} \frac{C_{F}}{C_{A}} P_{q g}(\zeta)\right) \ln \frac{\vec{k}^{2}}{\mu_{F}^{2}}-2 \zeta^{-2 \gamma} \ln R\left(P_{g g}(\zeta)+2 n_{f} P_{q g}(\zeta)\right)-\frac{\beta_{0}}{2} \ln \frac{\vec{k}^{2}}{4 \mu_{R}^{2}} \delta(1-\zeta)\right. \\
& +C_{A} \delta(1-\zeta)\left(\chi(n, \gamma) \ln \frac{s_{0}}{\vec{k}^{2}}+\frac{1}{12}+\frac{\pi^{2}}{6}+\frac{1}{2}\left(\psi^{\prime}\left(1+\gamma+\frac{n}{2}\right)-\psi^{\prime}\left(\frac{n}{2}-\gamma\right)-\chi^{2}(n, \gamma)\right)\right) \\
& +2 C_{A}\left(1-\zeta^{-2 \gamma}\right)\left(\left(\frac{1}{\zeta}-2+\zeta \bar{\zeta}\right) \ln \bar{\zeta}+\frac{\ln (1-\zeta)}{1-\zeta}\right) \\
& +C_{A}\left[\frac{1}{\zeta}+\frac{1}{(1-\zeta)_{+}}-2+\zeta \bar{\zeta}\right]\left(\left(1+\zeta^{-2 \gamma}\right) \chi(n, \gamma)-2 \ln \zeta+\frac{\bar{\zeta}^{2}}{\zeta^{2}} I_{2}\right) \\
& \left.\left.+n_{f}\left[2 \zeta \bar{\zeta} \frac{C_{F}}{C_{A}}+\left(\zeta^{2}+\bar{\zeta}^{2}\right)\left(\frac{C_{F}}{C_{A}} \chi(n, \gamma)+\frac{\bar{\zeta}}{\zeta} I_{3}\right)-\frac{1}{12} \delta(1-\zeta)\right]\right]\right\} \\
& c_{2}^{(1)}(n, \nu,|\vec{k}|, x)=\left[c_{1}^{(1)}(n, \nu,|\vec{k}|, x)\right]^{*} .
\end{aligned}
$$


Here $\bar{\zeta}=1-\zeta, \gamma=i \nu-1 / 2, P_{i j}(\zeta)$ are leading order DGLAP kernels. For the $I_{1,2,3}$ functions we have the results:

$$
\begin{gathered}
I_{2}=\frac{\zeta^{2}}{\bar{\zeta}^{2}}\left[\zeta\left(\frac{{ }_{2} F_{1}\left(1,1+\gamma-\frac{n}{2}, 2+\gamma-\frac{n}{2}, \zeta\right)}{\frac{n}{2}-\gamma-1}-\frac{{ }_{2} F_{1}\left(1,1+\gamma+\frac{n}{2}, 2+\gamma+\frac{n}{2}, \zeta\right)}{\frac{n}{2}+\gamma+1}\right)\right. \\
+\zeta^{-2 \gamma}\left(\frac{{ }_{2} F_{1}\left(1,-\gamma-\frac{n}{2}, 1-\gamma-\frac{n}{2}, \zeta\right)}{\frac{n}{2}+\gamma}-\frac{{ }_{2} F_{1}\left(1,-\gamma+\frac{n}{2}, 1-\gamma+\frac{n}{2}, \zeta\right)}{\frac{n}{2}-\gamma}\right) \\
\left.+\left(1+\zeta^{-2 \gamma}\right)(\chi(n, \gamma)-2 \ln \bar{\zeta})+2 \ln \zeta\right], \\
I_{1}=\frac{\bar{\zeta}}{2 \zeta} I_{2}+\frac{\zeta}{\bar{\zeta}}\left[\ln \zeta+\frac{1-\zeta^{-2 \gamma}}{2}(\chi(n, \gamma)-2 \ln \bar{\zeta})\right], \\
I_{3}=\frac{\bar{\zeta}}{2 \zeta} I_{2}-\frac{\zeta}{\bar{\zeta}}\left[\ln \zeta+\frac{1-\zeta^{-2 \gamma}}{2}(\chi(n, \gamma)-2 \ln \bar{\zeta})\right] .
\end{gathered}
$$

The factor $\zeta^{-\bar{\alpha}_{s}\left(\mu_{R}\right) \chi(n, \nu)}$ appears in (36) due to extra-contributions attributed to the jet vertices, as discussed after Eq. (29). Note that the $\mathcal{C}_{n}$ coefficients do not depend on the azimuthal angles of the jets, $\phi_{J_{1}}$ and $\phi_{J_{2}}$,

$$
\mathcal{C}_{n}=\mathcal{C}_{n}\left(y_{J_{1}}, y_{J_{2}}, \vec{k}_{J_{1}}, \vec{k}_{J_{2}}, \mu_{R}, \mu_{F}, s_{0}\right)
$$

they depend instead on the jet rapidities, the transverse momenta and on the factorization, renormalization and energy scale parameters.

An alternative way to present the differential cross section, equivalent to the formula (33) in the NLA, is the so-called exponentiated form (see Ref. [20]),

$$
\begin{gathered}
\mathcal{C}_{n}^{\exp }=\frac{x_{J_{1}} x_{J_{2}}}{\left|\vec{k}_{J_{1}}\right|\left|\vec{k}_{J_{2}}\right|} \int_{-\infty}^{+\infty} d \nu \exp \left[( Y - Y _ { 0 } ) \left(\bar{\alpha}_{s}\left(\mu_{R}\right) \chi(n, \nu)\right.\right. \\
\left.\left.+\bar{\alpha}_{s}^{2}\left(\mu_{R}\right)\left(\bar{\chi}(n, \nu)+\frac{\beta_{0}}{8 C_{A}} \chi(n, \nu)\left(-\chi(n, \nu)+\frac{10}{3}+\ln \frac{\mu_{R}^{4}}{\vec{k}_{J_{1}}^{2} \vec{k}_{J_{2}}^{2}}\right)\right)\right)\right] \\
\times \alpha_{s}^{2}\left(\mu_{R}\right) c_{1}\left(n, \nu,\left|\vec{k}_{J_{1}}\right|, x_{J_{1}}, \mu_{F}\right) c_{2}\left(n, \nu,\left|\vec{k}_{J_{2}}\right|, x_{J_{2}}, \mu_{F}\right) \\
\times\left[1+\alpha_{s}\left(\mu_{R}\right)\left(\frac{c_{1}^{(1)}\left(n, \nu,\left|\vec{k}_{J_{1}}\right|, x_{J_{1}}, \mu_{F}\right)}{c_{1}\left(n, \nu,\left|\vec{k}_{J_{1}}\right|, x_{J_{1}}, \mu_{F}\right)}+\frac{c_{2}^{(1)}\left(n, \nu,\left|\vec{k}_{J_{2}}\right|, x_{J_{2}}, \mu_{F}\right)}{c_{2}\left(n, \nu,\left|\vec{k}_{J_{2}}\right|, x_{J_{2}}, \mu_{F}\right)}\right)\right],
\end{gathered}
$$

where $Y$ is defined in Eq. (4) and we have introduced the variable

$$
Y_{0}=\ln \left(\frac{s_{0}}{\left|\vec{k}_{J_{1}}\right|\left|\vec{k}_{J_{2}}\right|}\right) \text {. }
$$

Below we will discuss the differential cross section integrated over the jet azimuthal angles

$$
\mathcal{C}_{0}=\int d \phi_{J_{1}} d \phi_{J_{2}} d \sigma
$$


the coefficients $\mathcal{C}_{n}$ and the moments of the azimuthal decorrelations, which are defined as

$$
\langle\cos (n \phi)\rangle=\frac{\int d \phi_{J_{1}} d \phi_{J_{2}} \cos \left[n\left(\phi_{J_{1}}-\phi_{J_{2}}-\pi\right)\right] d \sigma}{\int d \phi_{J_{1}} d \phi_{J_{2}} d \sigma}=\frac{\mathcal{C}_{n}}{\mathcal{C}_{0}} .
$$

\section{Results}

In this section we present our results for the dependence on $Y$ of the functions $\mathcal{C}_{n}$. In what follows we take the factorization and renormalization scales equal to each other, $\mu_{F}=\mu_{R}$. We perform our calculation both in the LLA and in the NLA. In the former case, the expression for $\mathcal{C}_{n}$ reads

$$
\begin{gathered}
\mathcal{C}_{n}^{\mathrm{LLA}}=\frac{d \sigma_{\mathrm{LLA}}}{d y_{J_{1}} d y_{J_{2}} d\left|\vec{k}_{J_{1}}\right| d\left|\vec{k}_{J_{2}}\right|}=\frac{x_{J_{1}} x_{J_{2}}}{\left|\vec{k}_{J_{1}}\right|\left|\vec{k}_{J_{2}}\right|} \int_{-\infty}^{+\infty} d \nu \exp \left[\left(Y-Y_{0}\right) \bar{\alpha}_{s}\left(\mu_{R}\right) \chi(n, \nu)\right] \\
\times \alpha_{s}^{2}\left(\mu_{R}\right) c_{1}\left(n, \nu,\left|\vec{k}_{J_{1}}\right|, x_{J_{1}}\right) c_{2}\left(n, \nu,\left|\vec{k}_{J_{2}}\right|, x_{J_{2}}\right) .
\end{gathered}
$$

For our NLA analysis we use the exponentiated representation given in Eq. (42).

For the center-of-mass energy $\sqrt{s}$ we take the LHC design value $14 \mathrm{TeV}$. We fix the jet cone size at the value $R=0.5$, in order to compare our predictions with the forthcoming LHC data. We study Mueller-Navelet jets with symmetric values of the transverse momenta, in particular, consider the choices: $\left|\vec{k}_{J_{1}}\right|=\left|\vec{k}_{J_{2}}\right|=35 \mathrm{GeV}$ and $\left|\vec{k}_{J_{1}}\right|=\left|\vec{k}_{J_{2}}\right|=20 \mathrm{GeV}$.

Moreover, to make possible the comparison with the experiments at present LHC energy, we perform calculations for $\sqrt{s}=7 \mathrm{TeV}$, where we consider the $\left|\vec{k}_{J_{1}}\right|=\left|\vec{k}_{J_{2}}\right|=35 \mathrm{GeV}$ case.

Following a quite recent CMS study [23], we restrict the rapidities of the Mueller-Navelet jets to the region $3 \leq\left|y_{J}\right| \leq 5$. We will present our results for $\mathcal{C}_{0}$, i.e. the differential cross section integrated over the jet azimuthal angles, the coefficients $\mathcal{C}_{n}$ for $n \neq 0$, and $\langle\cos (n \phi)\rangle$ versus the relative rapidity, $Y=y_{J_{1}}-y_{J_{2}}$. For our choice of forward jets rapidities, $Y$ takes values between 6 and 10. Our approach is similar to the one used in [10], we introduce rapidity bins with step $\Delta y_{J}$ equal to 0.5 , so the considered values for jet rapidities and rapidity difference are

$$
\begin{aligned}
& \left\{\left(y_{J_{1}}\right)_{i}\right\}=\{3.0,3.5,4.0,4.5,5.0\} \\
& \left\{\left(y_{J_{2}}\right)_{i}\right\}=\{-3.0,-3.5,-4.0,-4.5,-5.0\}
\end{aligned}
$$

and $\left\{Y_{i}\right\}=\{6.0,6.5,7.0,7.5,8.0,8.5,9.0,9.5,10.0\}$. Then we evaluate the following sum

$$
C_{n}\left(Y_{i}\right)=\sum 0.5 \mathcal{C}_{n}\left(\left(y_{J_{1}}\right)_{j},\left(y_{J_{1}}\right)_{j}-Y_{i}\right)
$$

where the sum runs over the possible values of $\left(y_{J_{1}}\right)_{j}$ for a given $Y_{i}$.

In our analysis we use the PDF set MSTW2008nnlo [24] and the two-loop running coupling with $\alpha_{s}\left(M_{Z}\right)=0.11707$. 
Our predictions depend on the values of energy and renormalization scales, $s_{0}$ and $\mu_{R}$. For the analysis in the LLA we fixed the values of these scales, $\mu_{R}$ and $s_{0}$, as suggested by the kinematics of the process, i.e. $\mu_{R}^{2}=s_{0}=\left|\vec{k}_{J_{1}}\right|\left|\vec{k}_{J_{2}}\right|$.

In general LLA results depend very strongly on $s_{0}$ and $\mu_{R}$, and one really needs to proceed to the NLA analysis in order to reduce this scale dependence and to have some reliable predictions for observables. One should stress that the dependence of the correlations $C_{n}$ on the scales $\mu_{R}$ and $s_{0}$ cancels with NLA accuracy; nevertheless in both representations (33) and (42) there unavoidably exist contributions subleading to NLA, depending on $\mu_{R}$ and $s_{0}$, whose numerical impact is important for the considered kinematics, therefore we need some prescription for the choice of these scales.

Following Ref. [20], we use here an adaptation of the principle of minimal sensitivity (PMS) [25, which consists in taking as optimal choices for $\mu_{R}$ and $s_{0}$ those values for which the physical observable under examination exhibits the minimal sensitivity to changes of both of these scales. The motivation of this procedure is that the complete resummation of the perturbative series would not depend on the scales $\mu_{R}$ and $s_{0}$, so the optimization method is supposed to mimic the effect of the most relevant unknown subleading terms.

In our search for optimal values, we took integer values for $Y_{0}$ in the range $0-6$ and values for $\mu_{R}$ given as integer multiples of $\sqrt{\left|\vec{k}_{J_{1}}\right|\left|\vec{k}_{J_{2}}\right|}$,

$$
\mu_{R}=n_{R} \sqrt{\left|\vec{k}_{J_{1}}\right|\left|\vec{k}_{J_{2}}\right|}
$$

taking the integer $n_{R}$ in the range $1-71$. The systematic uncertainty of the optimization procedure in the determination of observables, which will be discussed below, originates from the resolution of the grid in the $n_{R}-Y_{0}$ plane. This uncertainty has been estimated as the standard deviation of the optimal value from the determinations in the nearest neighbors of the grid. The error bars around the NLA data points presented in the figures below represent this uncertainty. We did not evaluate the impact on our predictions of the PDF uncertainties, since we expect it to be of the same size as determined in Ref. [10, where the same PDF set adopted here is used.

Let us start with the cross section integrated over the jet azimuthal angles, $C_{0}$. We found that for this observable a stationary point in the $n_{R}-Y_{0}$ plane could always be singled out, typically a local maximum. For $\sqrt{s}=14 \mathrm{TeV}$ our results, in $\left[\frac{\mathrm{nb}}{\mathrm{GeV}^{2}}\right]$ units, are presented in Figs. 2 3 and in Tables 1 2, results for $\sqrt{s}=7 \mathrm{TeV}$ are given in Fig. 4 and Table 3. The optimal values of $Y_{0}$ and $n_{R}=\mu_{R} / \sqrt{\left|\vec{k}_{J_{1}}\right|\left|\vec{k}_{J_{2}}\right|}$ are also reported in the tables. As in previous works [20], the optimal values of the energy scales turn to be a bit far from the kinematic scale. On the other hand, the uncertainty related of our optimization procedure, described above, turns out to be small, therefore our NLA results for the cross section integrated over jet azimuthal angles, presented in Figs. 24 have relatively small "error bars".

\footnotetext{
${ }^{1}$ Except than in a few cases (see Tables 1 , 6] and 7) always related with the approximate expressions combining LO impact factors and NLA Green's function, which we considered only for comparative purposes (see the text below).
} 
Similar considerations can be done for the observables $C_{1}$ and $C_{2}$ at $\sqrt{s}=14 \mathrm{TeV}$, to which we refer in Figs. 56 and in Tables 45 .

The other issue we addressed is the analysis of the observables $C_{1} / C_{0}, C_{2} / C_{0}$, which encode the first two non-trivial angular decorrelations: $\langle\cos \phi\rangle$ and $\langle\cos (2 \phi)\rangle$. For $\left|\vec{k}_{J_{1}}\right|=\left|\vec{k}_{J_{2}}\right|=35$ $\mathrm{GeV}$ at $\sqrt{s}=14 \mathrm{TeV}$ our results are presented in Fig. 7 and Tables [6 and 7 . For the smaller values of jet transverse momenta, $\left|\vec{k}_{J_{1}}\right|=\left|\vec{k}_{J_{2}}\right|=20 \mathrm{GeV}$, they are given in Fig. 8 and Table 8 ,

In this case, however, we were not able to find clear regions of stability in the $n_{R}-Y_{0}$ plane, therefore the results we present are obtained indirectly by using the optimal results for the observables $C_{0}, C_{1}$ and $C_{2}$.

Even if in general the energy dependence of the cross section and the azimuthal decorrelations is driven mainly by the kernel, in the considered kinematics the contribution of the NLO corrections to impact factors happened to be important. In order to show this, in the analysis at $\left|\vec{k}_{J_{1}}\right|=\left|\vec{k}_{J_{2}}\right|=35 \mathrm{GeV}$ and $\sqrt{s}=14 \mathrm{TeV}$ we calculated the coefficient $C_{0}$ and the correlations $C_{1} / C_{0}$ and $C_{2} / C_{0}$ using the NLA BFKL kernel together with the LO impact factors (properly modified with the inclusion of the NLO terms which guarantee the cancellation of the $\mu_{R^{-}}$and $s_{0}$-dependence in the NLA expressions for the coefficients $C_{n}$, see Ref. [26]). We find that the NLO corrections to impact factors are relevant especially at large values of Y, as shown in Figs. 2, 5 and 7 .

In the following Section we discuss the results presented here, make some comparison with Refs. [10, 11] and draw our conclusions.

\section{Discussion}

In this paper we considered in NLA BFKL approach the Mueller-Navelet jet production in proton-proton collisions, using the results for NLA jet vertices obtained recently in the "small-cone" approximation. Having a simple analytic result for the jet vertices, projected on the eigenfunction of LLA BFKL equation $((\nu, n)$-representation), one can implement them easily in the calculation of the Mueller-Navelet jet cross section. All necessary formulae are presented in Section 2.

We confirm the observation found earlier in the works devoted to forward electroproduction of a pair of vector mesons [20], and to Mueller-Navelet jets production [10], that NLA corrections to the impact factors (jet vertices) are very important and can not be ignored in a consistent NLA BFKL analysis.

Our numerical results, presented in Section 3, depend on the energy and renormalization scales, $s_{0}$ and $\mu_{R}$. The dependence of the coefficients $C_{n}$ on these scales cancels with NLA accuracy after the inclusion of NLA corrections to jet vertices. Nevertheless, due to next-toNLA contributions depending on $\mu_{R}$ and $s_{0}$, the observables we calculated are sensitive to the choice of these scales. Here, following Ref. [20, we used an optimization procedure, based on the principle of minimal sensitivity [25], which consists in taking as optimal choices for 
$\mu_{R}$ and $s_{0}$ those values for which the physical observable exhibits the minimal sensitivity to changes of both these scales.

The small-cone approximation, which we adopted here, is expected to be an adequate tool. Indeed, it is known that in the general case the dependence of the cross section on the jet cone parameter has, in the limit $R \rightarrow 0$, the form $d \sigma \sim A \ln R+B+\mathcal{O}\left(R^{2}\right)$ (see, for instance, [16] and Appendix $\mathrm{C}$ there). Indeed, in SCA the coefficients $A$ and $B$ are evaluated exactly. The neglected pieces $\mathcal{O}\left(R^{2}\right)$ for typical $R$ values are presumably less important than the other uncertainties of our NLA BFKL calculation, in particular those related with the choice of the scales $\mu_{R}$ and $s_{0}$, which mimic in our method the effect of the most relevant unknown next-to-NLA BFKL terms.

To support this statement it seems natural to make a comparison of our results obtained in SCA with the numerics presented in [10, 11], where the jet cone size was treated exactly. We would make such comparison for $\left|\vec{k}_{J_{1}}\right|=\left|\vec{k}_{J_{2}}\right|=35 \mathrm{GeV}$ and $\sqrt{s}=7$ and $14 \mathrm{TeV}$. For this purpose we present in Table 9 our NLA results obtained with the above-discussed optimal scales setting (presented in the third, fifth and seventh columns) and compare them with those for $C_{0},\langle\cos \phi\rangle$ and $\langle\cos (2 \phi)\rangle$ taken from Tables 1, 5 and 9 of Ref. [10] and reported in the second, fourth and sixth columns of our Table 9). Moreover, in Table 10 we show our NLA results in the case when kinematic values of the scales were used, $\mu_{R}^{2}=s_{0}=\left|\vec{k}_{J_{1}}\right|\left|\vec{k}_{J_{2}}\right|$.

Let us discuss the numbers presented in Tables 9 and 10. Firstly one needs to say that NLA results obtained with our formulae at kinematic scale setting, $\mu_{R}^{2}=s_{0}=\left|\vec{k}_{J_{1}}\right|\left|\vec{k}_{J_{2}}\right|$, can not be regarded as acceptable predictions for high values of $Y$. In particular we obtained even a negative value for the integrated cross section $C_{0}$ in the case of the largest rapidity difference, $Y=10$. This is related to the fact that NLO corrections to the jet vertices are negative and very large in absolute value when the kinematic scale setting is used. A similar observation was done in Refs. [20], where the electroproduction of a pair of vector mesons was considered. This is an indication of the fact that we actually need the PMS procedure in order to make reliable predictions. For $Y<9$ we can see a quite good agreement for $C_{0}$ with the results of Ref. [10], with a discrepancy rising with $Y$. As regards the moments of azimuthal decorrelation, we can see from Table 10 that for the kinematic scales our results for $Y=6$ agree with Ref. [10], and also here the discrepancy rises with $Y$. For the highest value of the rapidity separation, being $C_{0}$ negative at the kinematic scales, it makes no sense to report them in the table.

For the integrated cross section, $C_{0} 2$ the results presented in the second and third columns of Table 9 show the same trend, with a numerical discrepancy of about $15 \%$. This is due to the fact that in our approach, with higher values of the scales determined by the PMS optimization, we effectively take into account part of the subleading contributions, which are expected to be positive.

\footnotetext{
${ }^{2}$ Our LLA results coincide with those of [10] with high accuracy. We note, in passing, that the results quoted in Tables 15, 19, 35, 38, 53 and 56 of Ref. [10, giving the coefficients $C_{1}$ and $C_{2}$ for several values of the jet kinematics, should be multiplied by a factor two to correctly reproduce the values of the ratios $C_{1} / C_{0}$ and $C_{2} / C_{0}$ quoted in other tables of that paper. We stress that this normalization problem does not affect any of the comparisons presented in this work between our results and those of Ref. [10].
} 
Let us discuss now the observable $\langle\cos \phi\rangle$. Our predictions for it in the case of $\left|\vec{k}_{J_{1}}\right|=$ $\left|\vec{k}_{J_{2}}\right|=35 \mathrm{GeV}$ and $\sqrt{s}=14 \mathrm{TeV}$ are shown in Fig. 7 (left). In Table 9 we compare them (fourth and fifth columns) with those obtained in Ref. [10. Our results show a clear tendency for $\langle\cos \phi\rangle$ to decrease with $Y$, whereas [10] predicts a flat $Y$-dependence of $\langle\cos \phi\rangle$. A possible explanation of this discrepancy, as pointed our in a very recent paper [27], could be the different treatment of next-to-NLA corrections, which are beyond the precision of both studies.

Instead, for the observable $\langle\cos (2 \phi)\rangle$ the agreement between two approaches turns to be rather good, as sees in Table 9, sixth and seventh columns.

Similarly, we compare our predictions for $C_{0}$ at the present LHC energy $\sqrt{s}=7 \mathrm{TeV}$ with those of Ref. [11]. The agreement is rather fair, with a discrepancy of about 10\%, except for the case $Y=10$, as shown in Table 11 .

Traditionally the BFKL predictions are assumed to be compared with the fixed order DGLAP ones, trying to find a kinematic range where possible experiment can discriminate between these two approaches. For Mueller-Navelet process the relevant parameter which can describe the separation of these two regimes is $\eta=\bar{\alpha}_{s}\left(\left|\vec{k}_{J}\right|\right) Y$, which has the meaning of the mean number of hard undetected partons inclusively produced in the process. For $\left|\vec{k}_{J}\right|=35 \mathrm{GeV}$ and $Y=6 \div 10$ this parameter takes the values $\eta=0.82 \div 1.37$. In Section 3 we presented also our predictions for smaller jets transverse momenta, $\left|\vec{k}_{J}\right|=20 \mathrm{GeV}$, where $\eta=0.92 \div 1.53$ for $Y=6 \div 10$. In this case the BFKL description is expected to give results more different with respect to the NLO DGLAP ones. We hope that experiments with such Mueller-Navelet jet transverse momenta will be possible in the future at LHC.

Our PMS optimization procedure for the kinematics considered gives us optimal energy and factorization scales values which are substantially larger than the scale given by the kinematics, $\mu_{R}^{2}=\left|\vec{k}_{J_{1}}\right|\left|\vec{k}_{J_{2}}\right|$, especially for higher values of $Y$. This fact indicates the presence of important contributions subleading to the NLA. Therefore the estimates of the uncertainties in our predictions should be taken with care. Nevertheless they reflect the reliability of PMS method in the considered kinematics. Note that despite very large negative contributions to the MN-jet cross section coming both from the NLA corrections to the BFKL kernel and NLA corrections to the jet vertices with respect to LLA MN-jet cross section, we got with our PMS procedure rather precise results in all cases. We point out that this approach was unsatisfactory in the case of asymmetric kinematics, as already happened in previous studies [20]; in this kinematics it could be noteworthy to turn to approaches based on collinear improvement of the BFKL kernel [28, 29].

It would be very interesting to confront our predictions with experiment. However, we must mention that the numerical results we presented here refer to cross sections and angular decorrelations that are differential in the jet transverse momenta, whereas experimental data would inevitably include some bins in the transverse momenta of the jets. For wide bins in the jet transverse momentum, one definitely needs to perform a new numerical calculation with our formulas, but this goes beyond the scope of our present study.

At the very end let us comment a situation related with the kinematics when jets are basically back-to-back in the transverse plane (with transverse momenta set equal $\left|\vec{k}_{J_{1}}\right|=\left|\vec{k}_{J_{2}}\right|$ 
or very close to each other). It has been known for some time [30] that in this kinematic range the standard NLO QCD collinear calculations for dijet production in electron-proton collisions exhibit some unreliable features. In particular, the NLO inclusive dijet total cross section for $\left|\vec{k}_{J_{1}}\right|>E_{\text {cut }}$ and $\left|\vec{k}_{J_{2}}\right|>E_{\text {cut }}+\Delta$, contrary to expectations based on simple phase-space considerations, does not exhibit a monotonic decrease for increasing $\Delta$, but has instead a local maximum at some small values of $\Delta, \Delta_{\max }\left(E_{\text {cut }}\right) \ll E_{\text {cut }}$. A similar feature of QCD NLO prediction was observed also for the production of dijets in proton-proton collisions in the case when a (nearly) symmetric restriction on the transverse momenta of the two jets is imposed, see [31, 32]. The qualitative explanation of such a failure of the NLO QCD approach (the dip in the cross section at $\Delta \rightarrow 0$ ) was suggested by the authors of [30] and is related to the fact that, by imposing on the two tagged jets back-to-back condition in the transverse momentum plane, one effectively puts a veto on the emission of a soft real gluon. This veto prevents the compensation of the large negative contribution coming from the soft-virtual term. In this case, an all-order resummation of soft-gluon effects is needed to get a consistent result. Such resummation was considered for electron-proton collision in [33] and it is incorporated in the POWHEG method [34, 35]. Having soft gluon resummation built in through the parton showering, the POWHEG predictions for dijet production in proton-proton collisions exhibit a monotonic decrease with $\Delta$, in contrast to the NLO case, see [32].

In the case of Mueller-Navelet jet production the situation with the above-mentioned symmetric transverse momentum jet tagging is different from the inclusive dijet total cross section. In Mueller-Navelet jet kinematics the tagged jets are separated for sure by a large rapidity interval $Y$, which reduces the price paid for the radiation of hard unidentified partons. Roughly speaking, each additional hard-parton radiation "costs" a factor $\sim \eta=\bar{\alpha}_{s}\left(\left|\vec{k}_{J}\right|\right) Y$, being of order of unity for our kinematics. Note that after a single additional hard-parton emission the transverse momentum deposit in the event is essentially redistributed and the symmetric transverse momentum jet tagging condition $\left(\left|\vec{k}_{J_{1}}\right|>E_{\text {cut }}\right.$ and $\left.\left|\vec{k}_{J_{2}}\right|>E_{\text {cut }}\right)$ cannot play anymore the role of a veto for the subsequent emission of real gluons. In this case the compensation of large effects coming from the virtual and real emission of soft gluons is effective and one expects a small net effect in the sum of real and virtual soft gluon contributions.

\section{Acknowledgements}

We are grateful to the authors of Ref. [11] for providing us with the data reported in the second column of Table 11, We acknowledge also some stimulating discussions with Agustin Sabio Vera.

D.I. thanks the Dipartimento di Fisica dell'Università della Calabria and the Istituto Nazionale di Fisica Nucleare (INFN), Gruppo collegato di Cosenza, for the warm hospitality and the financial support. The work of D.I. was also supported in part by the grants and RFBR-11-02-00242 and NSh-3810.2010.2. The work of F.C. was supported by European Commission, European Social Fund and Calabria Region, that disclaim any liability for the use that can be done of the information provided in this paper. 


\section{References}

[1] A.H. Mueller, H. Navelet, Nucl. Phys. B 282 (1987) 727.

[2] V.S. Fadin, E.A. Kuraev, L.N. Lipatov, Phys. Lett. B60 (1975) 50; E.A. Kuraev, L.N. Lipatov and V.S. Fadin, Zh. Eksp. Teor. Fiz. 71 (1976) 840 [Sov. Phys. JETP 44 (1976) 443]; 72 (1977) 377 [45 (1977) 199]; Ya.Ya. Balitskii and L.N. Lipatov, Sov. J. Nucl. Phys. 28 (1978) 822.

[3] V.N. Gribov, L.N. Lipatov, Sov. J. Nucl. Phys. 15 (1972) 438; G. Altarelli, G. Parisi, Nucl. Phys. B 126 (1977) 298; Y.L. Dokshitzer, Sov. Phys. JETP 46 (1977) 641.

[4] V.S. Fadin, L.N. Lipatov, Phys. Lett. B429 (1998) 127.

[5] G. Camici and M. Ciafaloni, Phys. Lett. B430 (1998) 349.

[6] V.S. Fadin, R. Fiore and A. Papa, Phys. Rev. D 60 (1999) 074025.

[7] V.S. Fadin and D.A. Gorbachev, Pisma v Zh. Eksp. Teor. Fiz. 71 (2000) 322 [JETP Letters 71 (2000) 222]; Phys. Atom. Nucl. 63 (2000) 2157 [Yad. Fiz. 63 (2000) 2253].

[8] V.S. Fadin and R. Fiore, Phys. Lett. B610 (2005) 61 [Erratum-ibid. 621 (2005) 61]; Phys. Rev. D 72 (2005) 014018.

[9] J. Bartels, D. Colferai and G.P. Vacca, Eur. Phys. J. C 24 (2002) 83; Eur. Phys. J. C 29 (2003) 235;

[10] D. Colferai, F. Schwennsen, L. Szymanowski, S. Wallon, JHEP 1012 (2010) 026.

[11] B. Ducloué, L. Szymanowski and S. Wallon, PoS QNP 2012 (2012) 165 arXiv:1208.6111 [hep-ph]]; JHEP 1305 (2013) 096.

[12] A. Sabio Vera, Nucl. Phys. B 746 (2006) 1; A. Sabio Vera, F. Schwennsen, Nucl. Phys. B 776 (2007) 170.

[13] C. Marquet, C. Royon, Phys. Rev. D 79 (2009) 034028.

[14] F. Caporale, D.Yu. Ivanov, B. Murdaca, A. Papa and A. Perri, JHEP 1202 (2012) 101.

[15] D.Yu. Ivanov and A. Papa, JHEP 1205 (2012) 086.

[16] M. Furman, Nucl. Phys. B 197 (1982) 413.

[17] F. Aversa, P. Chiappetta, M. Greco, J.P. Guillet, Nucl. Phys. B 327 (1989) 105; Z. Phys. C 46 (1990) 253.

[18] S. Catani, M. Ciafaloni and F. Hautmann, Phys. Lett. B307 (1993) 147; Nucl. Phys. B 366 (1991) 135; Phys. Lett. B242 (1990) 97.

[19] M. Deak, F. Hautmann, H. Jung and K. Kutak, JHEP 0909 (2009) 121. 
[20] D.Yu. Ivanov and A. Papa, Nucl. Phys. B 732 (2006) 183; Eur. Phys. J. C 49 (2007) 947; F. Caporale, A. Papa and A. Sabio Vera, Eur. Phys. J. C 53 (2008) 525.

[21] A.V. Kotikov and L.N. Lipatov, Nucl. Phys. B 582 (2000) 19.

[22] A.V. Kotikov and L.N. Lipatov, Nucl. Phys. B 661 (2003) 19 [Erratum-ibid. B 685 (2004) 405].

[23] S. Cerci and D. d'Enterria, AIP Conf. Proc. 1105 (2009) 28.

[24] A.D. Martin, W.J. Stirling, R.S. Thorne and G. Watt, Eur. Phys. J. C 63 (2009) 189.

[25] P.M. Stevenson, Phys. Lett. B100 (1981) 61; Phys. Rev. D 23 (1981) 2916.

[26] F. Caporale, D.Yu. Ivanov and A. Papa, Eur. Phys. J. C 58 (2008) 1.

[27] B. Ducloué, L. Szymanowski and S. Wallon, JHEP 1305 (2013) 096.

[28] G.P. Salam, JHEP 9807 (1998) 019.

[29] A. Sabio Vera, Nucl. Phys. B 722 (2005) 65.

[30] S. Frixione and G. Ridolfi, Nucl. Phys. B 507 (1997) 315.

[31] J.R. Andersen, V. Del Duca, S. Frixione, C.R. Schmidt and W.J. Stirling, JHEP 0102 (2001) 007.

[32] S. Alioli, K. Hamilton, P. Nason, C. Oleari and E. Re, JHEP 1104 (2011) 081.

[33] A. Banfi and M. Dasgupta, JHEP 0401 (2004) 027.

[34] P. Nason, JHEP 0411 (2004) 040.

[35] S. Frixione, P. Nason and C. Oleari, JHEP 0711 (2007) 070. 


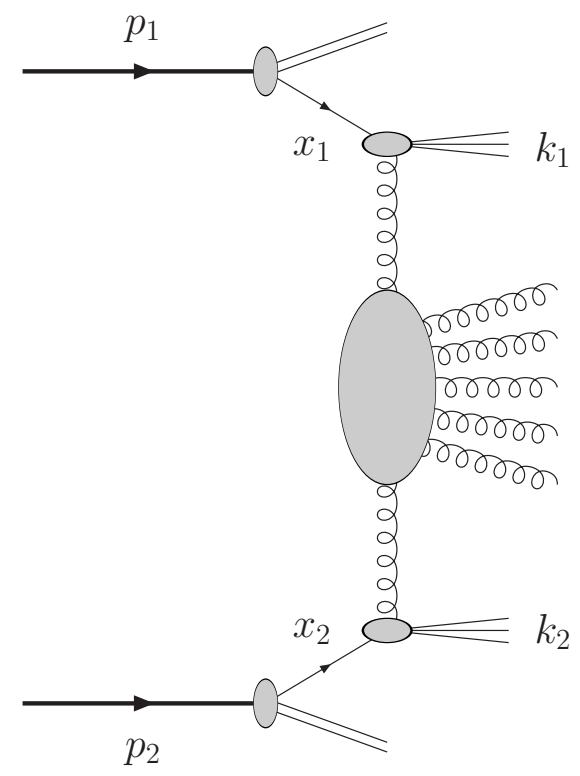

Figure 1: Mueller-Navelet jet production process.

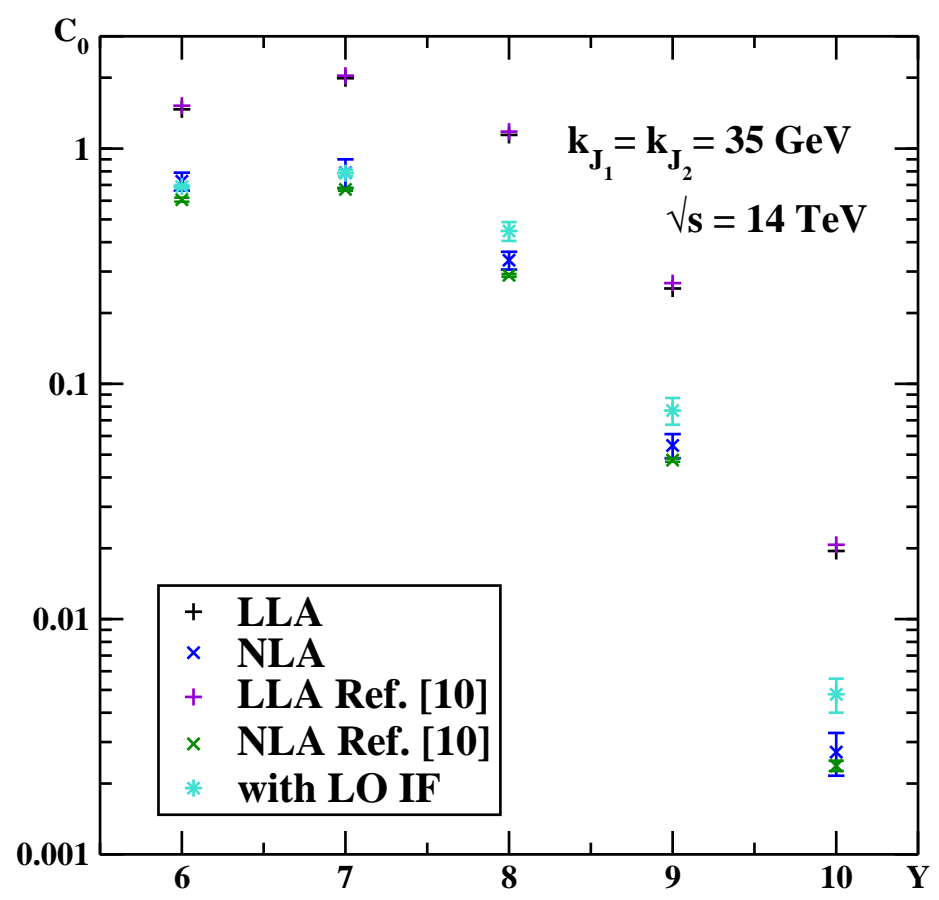

Figure 2: $Y$ dependence of $C_{0}$ for $\left|\vec{k}_{J_{1}}\right|=\left|\vec{k}_{J_{2}}\right|=35 \mathrm{GeV}$ at $\sqrt{s}=14 \mathrm{TeV}$. 


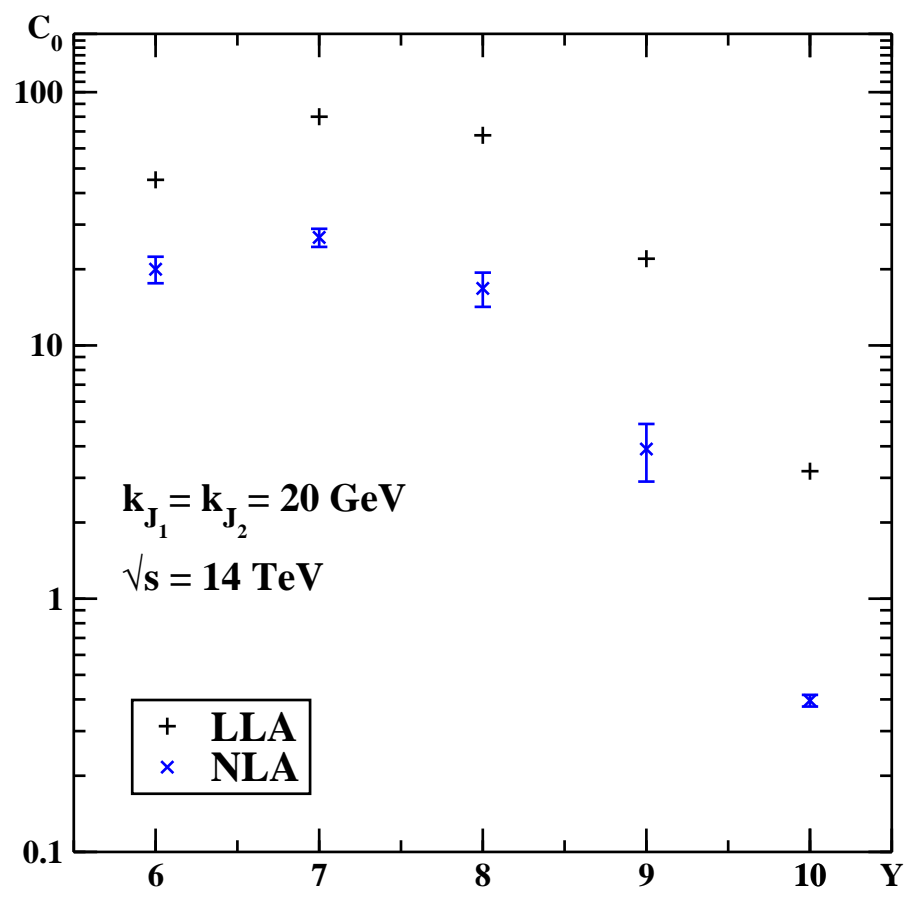

Figure 3: $Y$ dependence of $C_{0}$ for $\left|\vec{k}_{J_{1}}\right|=\left|\vec{k}_{J_{2}}\right|=20 \mathrm{GeV}$ at $\sqrt{s}=14 \mathrm{TeV}$.

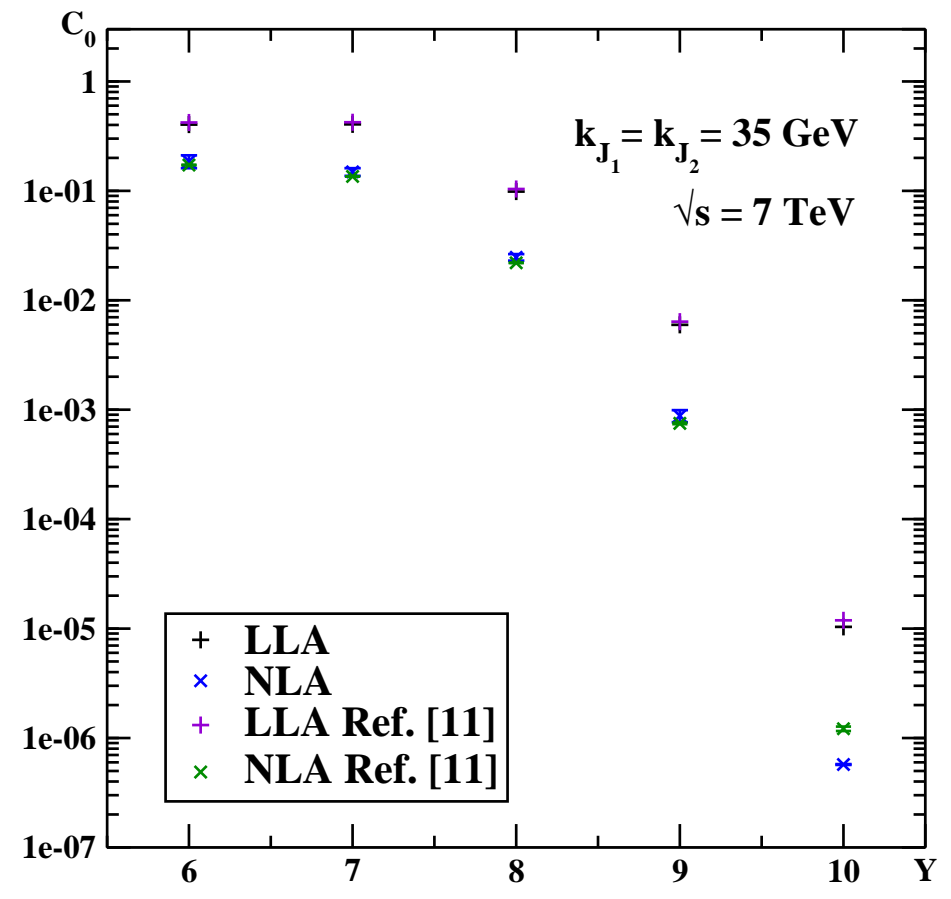

Figure 4: $Y$ dependence of $C_{0}$ for $\left|\vec{k}_{J_{1}}\right|=\left|\vec{k}_{J_{2}}\right|=35 \mathrm{GeV}$ at $\sqrt{s}=7 \mathrm{TeV}$. 

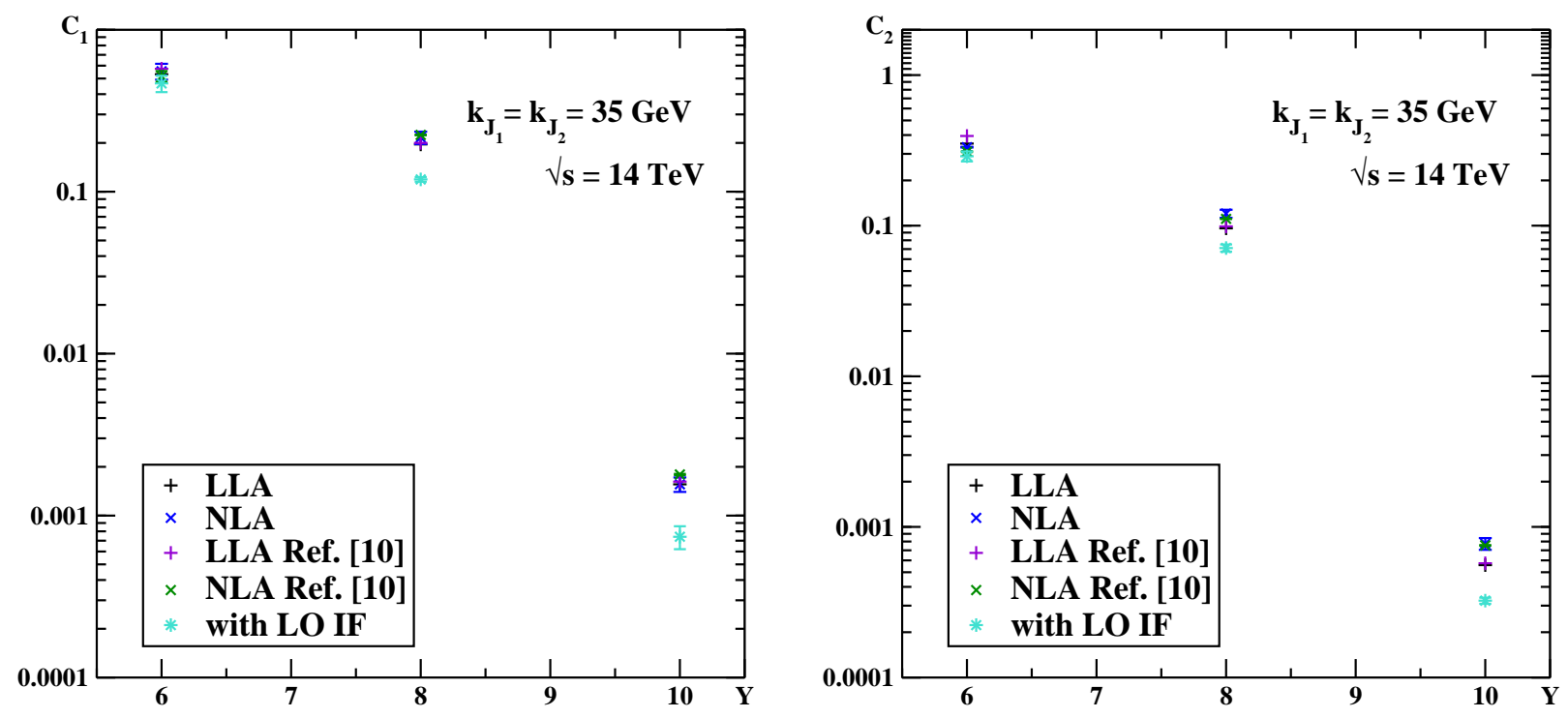

Figure 5: $Y$ dependence of $C_{1}$ (left) and $C_{2}$ (right) for $\left|\vec{k}_{J_{1}}\right|=\left|\vec{k}_{J_{2}}\right|=35 \mathrm{GeV}$ at $\sqrt{s}=14$ $\mathrm{TeV}$.
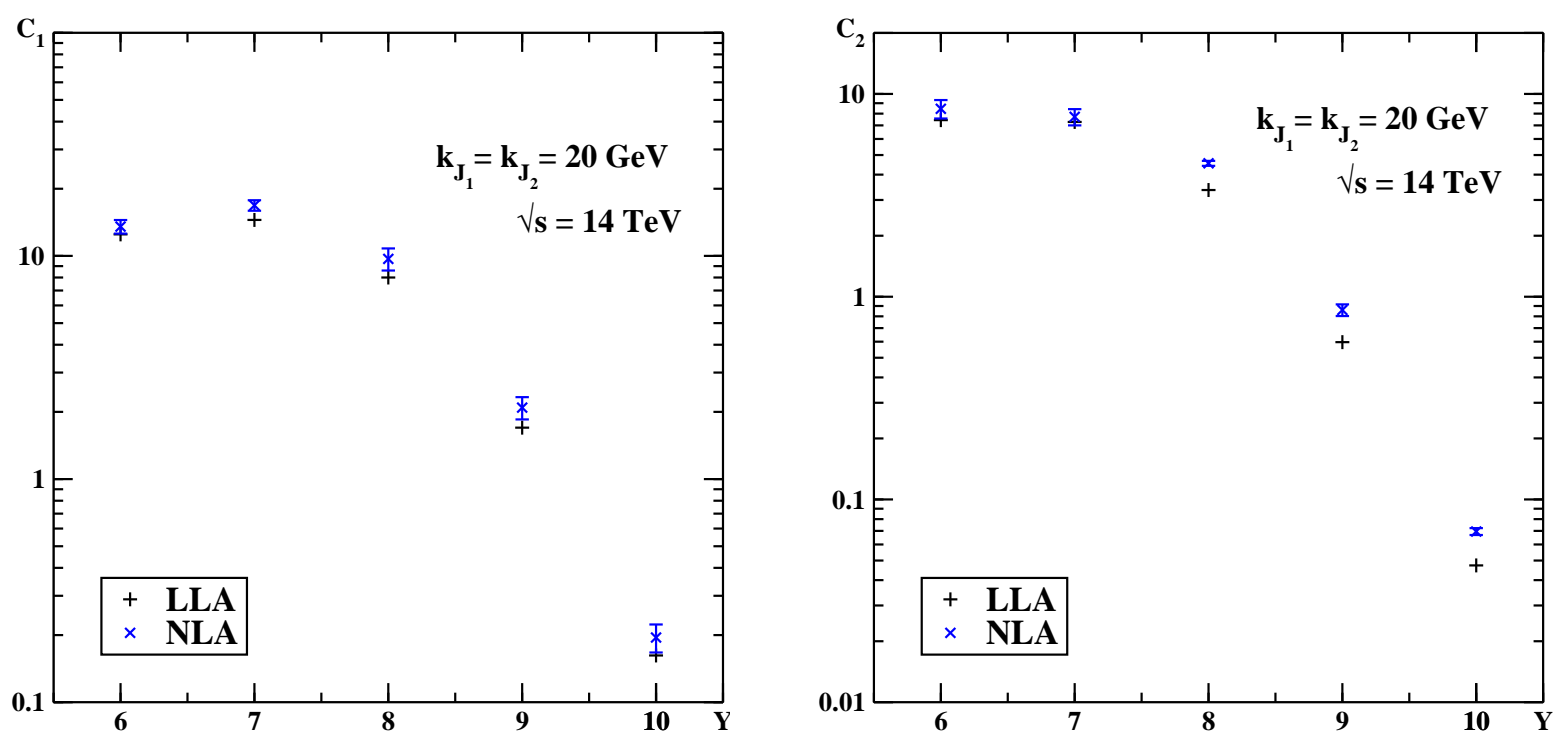

Figure 6: $Y$ dependence of $C_{1}$ (left) and $C_{2}$ (right) for $\left|\vec{k}_{J_{1}}\right|=\left|\vec{k}_{J_{2}}\right|=20 \mathrm{GeV}$ at $\sqrt{s}=14$ $\mathrm{TeV}$. 

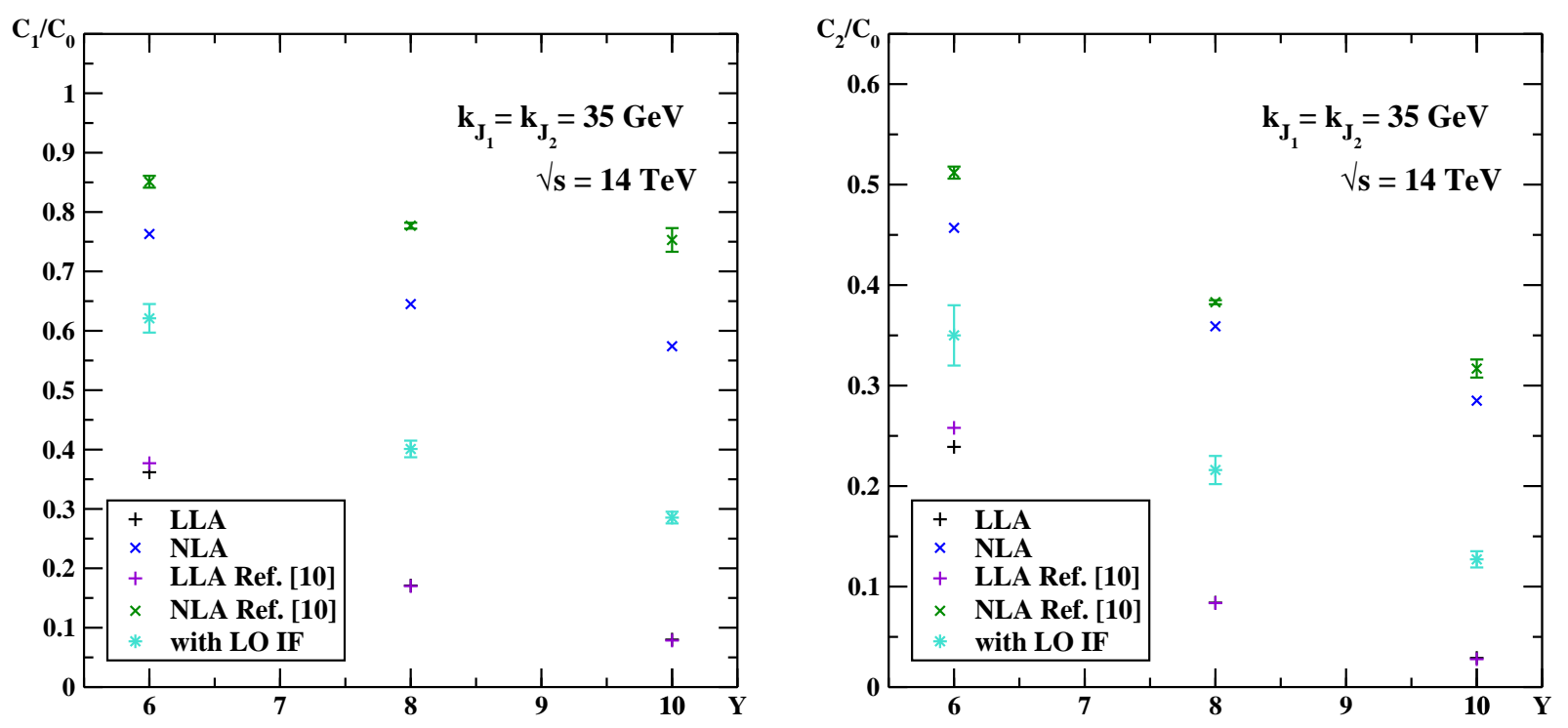

Figure 7: $Y$ dependence of $C_{1} / C_{0}$ (left) and $C_{2} / C_{0}$ (right) for $\left|\vec{k}_{J_{1}}\right|=\left|\vec{k}_{J_{2}}\right|=35 \mathrm{GeV}$ at $\sqrt{s}=14 \mathrm{TeV}$. Please note that LLA data at $Y=8$ and 10 from the present work and from Ref. [10] are barely distinguishable in these plots.
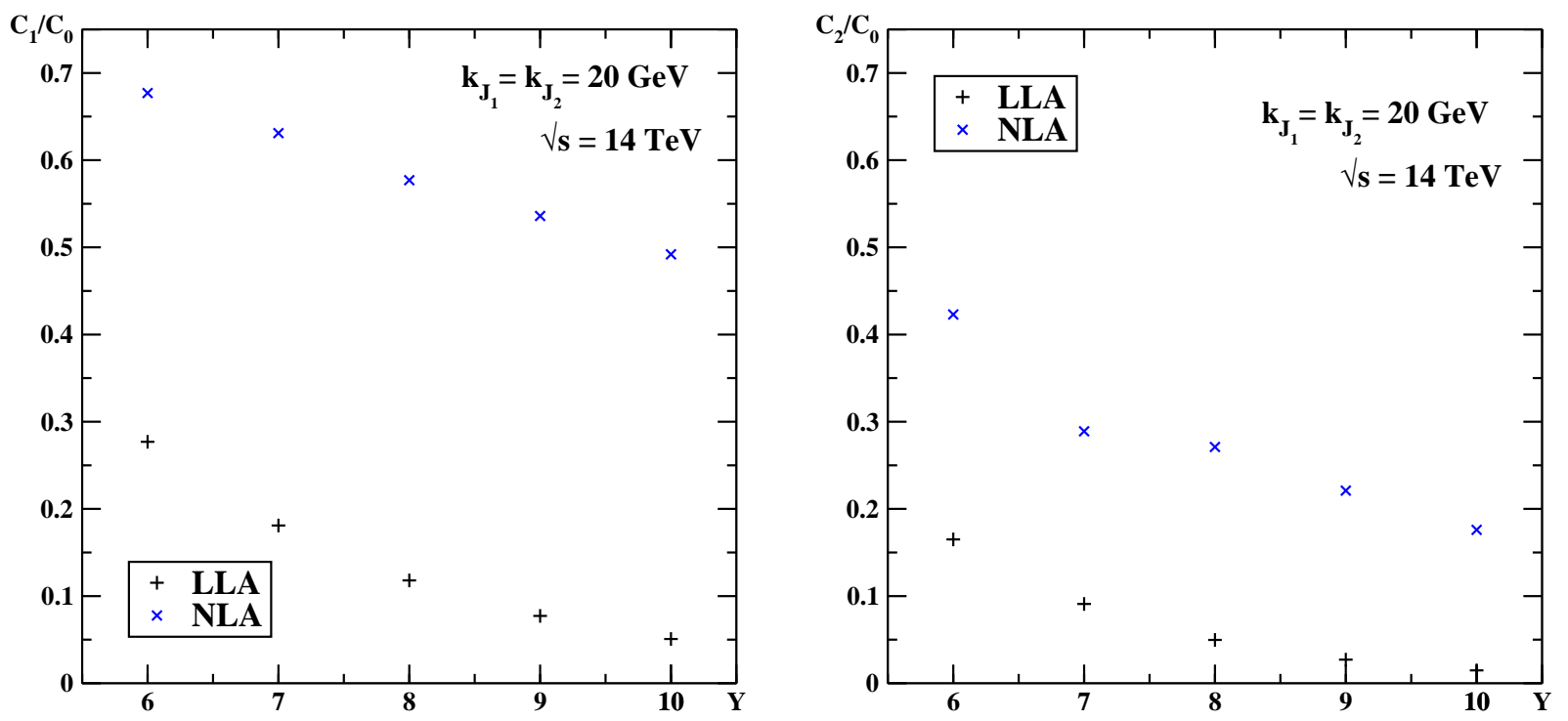

Figure 8: $Y$ dependence of $C_{1} / C_{0}$ (left) and $C_{2} / C_{0}$ (right) for $\left|\vec{k}_{J_{1}}\right|=\left|\vec{k}_{J_{2}}\right|=20 \mathrm{GeV}$ at $\sqrt{s}=14 \mathrm{TeV}$. 


\begin{tabular}{|r|c|c|c|c||c|c|c|}
\hline$Y$ & $C_{0}^{(\mathrm{LLA})}$ & $C_{0}^{(\mathrm{NLA})}$ & $Y_{0}$ & $n_{R}$ & $C_{0}^{(\mathrm{NLA} / \mathrm{LO} I \mathrm{IF})}$ & $Y_{0}$ & $n_{R}$ \\
\hline 6 & 1.468 & $0.726(64)$ & 1 & 2 & $0.689(11)$ & 1 & 12 \\
7 & 1.990 & $0.79(11)$ & 2 & 2 & $0.786(23)$ & 1 & 6 \\
8 & 1.142 & $0.335(29)$ & 2 & 2 & $0.446(41)$ & 1 & 2 \\
9 & 0.2542 & $0.0547(64)$ & 3 & 2 & $0.077(10)$ & 1 & 2 \\
10 & 0.01947 & $0.00272(56)$ & 4 & 2 & $0.00479(79)$ & 1 & 2 \\
\hline
\end{tabular}

Table 1: Values of $C_{0}$ in the LLA, in the NLA and in the NLA with LO impact factors for $\left|\vec{k}_{J_{1}}\right|=\left|\vec{k}_{J_{2}}\right|=35 \mathrm{GeV}$ at $\sqrt{s}=14 \mathrm{TeV}$, corresponding to the data points in Fig. 2, The optimal values of $Y_{0}$ and $n_{R}=\mu_{R} / \sqrt{\left|\vec{k}_{J_{1}}\right|\left|\vec{k}_{J_{2}}\right|}$ for $C_{0}^{(\mathrm{NLA})}$ are given in the fourth and fifth columns, while those for $C_{0}^{(\mathrm{NLA} / \mathrm{LO} \mathrm{IF})}$ are given in the last two columns.

\begin{tabular}{|r|c|c|c|c|}
\hline$Y$ & $C_{0}^{\text {(LLA) }}$ & $C_{0}^{\text {(NLA) }}$ & $Y_{0}$ & $n_{R}$ \\
\hline 6 & 45.12 & $20.0(24)$ & 1 & 1 \\
7 & 80.03 & $26.7(22)$ & 1 & 1 \\
8 & 67.65 & $16.8(26)$ & 2 & 1 \\
9 & 21.99 & $3.9(10)$ & 3 & 1 \\
10 & 3.187 & $0.396(21)$ & 3 & 2 \\
\hline
\end{tabular}

Table 2: Values of $C_{0}$ in the LLA and in the NLA for $\left|\vec{k}_{J_{1}}\right|=\left|\vec{k}_{J_{2}}\right|=20 \mathrm{GeV}$ at $\sqrt{s}=14 \mathrm{TeV}$, corresponding to the data points in Fig. 3. The last two columns give the optimal values of $Y_{0}$ and $n_{R}=\mu_{R} / \sqrt{\left|\vec{k}_{J_{1}}\right|\left|\vec{k}_{J_{2}}\right|}$.

\begin{tabular}{|r|c|c|c|c|}
\hline$Y$ & $C_{0}^{(\mathrm{LLA})}$ & $C_{0}^{(\mathrm{NLA})}$ & $Y_{0}$ & $n_{R}$ \\
\hline 6 & 0.403 & $0.186(25)$ & 2 & 2 \\
7 & 0.405 & $0.149(12)$ & 2 & 3 \\
8 & 0.0984 & $0.0247(17)$ & 3 & 3 \\
9 & 0.005974 & $0.00088(11)$ & 4 & 3 \\
10 & $103.6210^{-7}$ & $5.73(51) 10^{-7}$ & 5.5 & 7 \\
\hline
\end{tabular}

Table 3: Values of $C_{0}$ in the LLA and in the NLA for $\left|\vec{k}_{J_{1}}\right|=\left|\vec{k}_{J_{2}}\right|=35 \mathrm{GeV}$ at $\sqrt{s}=7 \mathrm{TeV}$, corresponding to the data points in Fig. 4. The last two columns give the optimal values of $Y_{0}$ and $n_{R}=\mu_{R} / \sqrt{\left|\vec{k}_{J_{1}}\right|\left|\vec{k}_{J_{2}}\right|}$. 


\begin{tabular}{|r|c|c|c|c||c|c|c|c|}
\hline$Y$ & $C_{1}^{\text {(LLA) }}$ & $C_{1}^{(\mathrm{NLA})}$ & $Y_{0}$ & $n_{R}$ & $C_{2}^{\text {(LLA) }}$ & $C_{2}^{(\mathrm{NLA})}$ & $Y_{0}$ & $n_{R}$ \\
\hline 6 & 0.531 & $0.554(62)$ & 1 & 2 & 0.351 & $0.3320(18)$ & 0 & 1.5 \\
8 & 0.196 & $0.216(19)$ & 2 & 2 & 0.0961 & $0.1203(74)$ & 2 & 2.5 \\
10 & 0.00156 & $0.00156(16)$ & 3 & 2 & 0.000558 & $0.000774(69)$ & 4 & 4 \\
\hline
\end{tabular}

Table 4: Values of $C_{1}$ and $C_{2}$ in the LLA and in the NLA for $\left|\vec{k}_{J_{1}}\right|=\left|\vec{k}_{J_{2}}\right|=35 \mathrm{GeV}$ at $\sqrt{s}=14 \mathrm{TeV}$, corresponding to the data points in Fig. 5. The optimal values of $Y_{0}$ and $n_{R}=\mu_{R} / \sqrt{\left|\vec{k}_{J_{1}}\right|\left|\vec{k}_{J_{2}}\right|}$ for $C_{1}^{(\mathrm{NLA})}$ are given in the fourth and fifth columns, while those for $C_{2}^{(\mathrm{NLA})}$ are given in the last two columns.

\begin{tabular}{|r|c|c|c|c||c|c|c|c|}
\hline$Y$ & $C_{1}^{\text {(LLA })}$ & $C_{1}^{\text {(NLA) }}$ & $Y_{0}$ & $n_{R}$ & $C_{2}^{\text {(LLA })}$ & $C_{2}^{\text {(NLA) }}$ & $Y_{0}$ & $n_{R}$ \\
\hline 6 & 12.49 & $13.54(95)$ & 0 & 1 & 7.43 & $8.45(88)$ & 1 & 3 \\
7 & 14.50 & $16.85(91)$ & 0.5 & 1 & 7.28 & $7.71(71)$ & 0.5 & 1 \\
8 & 8.0 & $9.7(11)$ & 1 & 1 & 3.36 & $4.55(13)$ & 2.5 & 4 \\
9 & 1.70 & $2.09(24)$ & 1 & 1 & 0.597 & $0.860(57)$ & 3 & 5 \\
10 & 0.162 & $0.195(28)$ & 1 & 1 & 0.0473 & $0.0695(27)$ & 3.5 & 3 \\
\hline
\end{tabular}

Table 5: Values of $C_{1}$ and $C_{2}$ in the LLA and in the NLA for $\left|\vec{k}_{J_{1}}\right|=\left|\vec{k}_{J_{2}}\right|=20 \mathrm{GeV}$ at $\sqrt{s}=14 \mathrm{TeV}$, corresponding to the data points in Fig. 6. The optimal values of $Y_{0}$ and $n_{R}=\mu_{R} / \sqrt{\left|\vec{k}_{J_{1}}\right|\left|\vec{k}_{J_{2}}\right|}$ for $C_{1}^{(\mathrm{NLA})}$ are given in the fourth and fifth columns, while those for $C_{2}^{(\mathrm{NLA})}$ are given in the last two columns. 


\begin{tabular}{|r|c|c||c|c|c|}
\hline$Y$ & $C_{1}^{(\mathrm{LLA})} / C_{0}^{(\mathrm{LLA})}$ & $C_{1}^{(\mathrm{NLA})} / C_{0}^{(\mathrm{NLA})}$ & $C_{1}^{\text {(NLA } / \mathrm{LO} \mathrm{IF)}} / C_{0}^{(\mathrm{NLA} / \mathrm{LO} I F)}$ & $Y_{0}$ & $n_{R}$ \\
\hline 6 & 0.3618 & 0.763 & $0.621(24)$ & 2 & 10 \\
8 & 0.171 & 0.645 & $0.401(14)$ & 2 & 5 \\
10 & 0.080 & 0.574 & $0.2854(98)$ & 3 & 5 \\
\hline
\end{tabular}

Table 6: Values of $C_{1} / C_{0}=\langle\cos \phi\rangle$ in the LLA, in the NLA and in the NLA with LO impact factors for $\left|\vec{k}_{J_{1}}\right|=\left|\vec{k}_{J_{2}}\right|=35 \mathrm{GeV}$ at $\sqrt{s}=14 \mathrm{TeV}$, corresponding to the data points in Fig. 7 (left). The optimal values of $Y_{0}$ and $n_{R}=\mu_{R} / \sqrt{\left|\vec{k}_{J_{1}}\right|\left|\vec{k}_{J_{2}}\right|}$ for $C_{1}^{(\mathrm{NLA} / \mathrm{LO} \text { IF) }} / C_{0}^{\text {(NLA/LO IF) }}$ are given in the last two columns.

\begin{tabular}{|r|c|c||c|c|c|}
\hline$Y$ & $C_{2}^{(\mathrm{LLA})} / C_{0}^{(\mathrm{LLA})}$ & $C_{2}^{(\mathrm{NLA})} / C_{0}^{(\mathrm{NLA})}$ & $C_{2}^{(\mathrm{NLA} / \mathrm{LO} I F)} / C_{0}^{(\mathrm{NLA} / \mathrm{LO} I F)}$ & $Y_{0}$ & $n_{R}$ \\
\hline 6 & 0.239 & 0.457 & $0.350(30)$ & 1 & 3 \\
8 & 0.084 & 0.359 & $0.216(14)$ & 2 & 7 \\
10 & 0.029 & 0.285 & $0.1271(81)$ & 3 & 12 \\
\hline
\end{tabular}

Table 7: Values of $C_{2} / C_{0}=\langle\cos (2 \phi)\rangle$ in the LLA, in the NLA and in the NLA with LO impact factors for $\left|\vec{k}_{J_{1}}\right|=\left|\vec{k}_{J_{2}}\right|=35 \mathrm{GeV}$ at $\sqrt{s}=14 \mathrm{TeV}$, corresponding to the data points in Fig. 7 (right). The optimal values of $Y_{0}$ and $n_{R}=\mu_{R} / \sqrt{\left|\vec{k}_{J_{1}}\right|\left|\vec{k}_{J_{2}}\right|}$ for $C_{2}^{(\mathrm{NLA} / \mathrm{LO} \mathrm{IF})} / C_{0}^{(\mathrm{NLA} / \mathrm{LO} \text { IF) }}$ are given in the last two columns.

\begin{tabular}{|r|c|c||c|c|}
\hline$Y$ & $C_{1}^{(\mathrm{LLA})} / C_{0}^{(\mathrm{LLA})}$ & $C_{1}^{(\mathrm{NLA})} / C_{0}^{(\mathrm{NLA})}$ & $C_{2}^{(\mathrm{LLA})} / C_{0}^{(\mathrm{LLA})}$ & $C_{2}^{(\mathrm{NLA})} / C_{0}^{(\mathrm{NLA})}$ \\
\hline 6 & 0.277 & 0.677 & 0.165 & 0.423 \\
7 & 0.181 & 0.631 & 0.091 & 0.289 \\
8 & 0.118 & 0.577 & 0.050 & 0.271 \\
9 & 0.077 & 0.536 & 0.027 & 0.221 \\
10 & 0.051 & 0.492 & 0.015 & 0.176 \\
\hline
\end{tabular}

Table 8: Values of $C_{1} / C_{0}=\langle\cos \phi\rangle$ and $C_{2} / C_{0}=\langle\cos (2 \phi)\rangle$ in the LLA and in the NLA for $\left|\vec{k}_{J_{1}}\right|=\left|\vec{k}_{J_{2}}\right|=20 \mathrm{GeV}$ at $\sqrt{s}=14 \mathrm{TeV}$, corresponding to the data points in Fig. 8 ,

\begin{tabular}{|c|c|c|c|c|c|c|}
\hline$Y$ & $\begin{array}{l}C_{0}^{(\mathrm{NLA})} \\
\text { [Ref. [10]] }\end{array}$ & $\begin{array}{l}C_{0}^{(\mathrm{NLA})} \\
\text { [here] }\end{array}$ & $\begin{array}{l}\langle\cos \phi\rangle^{\mathrm{NLA}} \\
\text { [Ref. [10]] }\end{array}$ & $\begin{array}{c}\langle\cos \phi\rangle^{\mathrm{NLA}} \\
\text { [here] }\end{array}$ & $\begin{array}{c}\langle\cos (2 \phi)\rangle^{\mathrm{NLA}} \\
{[\text { Ref. [10]] }}\end{array}$ & $\begin{array}{c}\langle\cos (2 \phi)\rangle^{\mathrm{NLA}} \\
\text { [here] }\end{array}$ \\
\hline 6 & 0.606 & 0.726 & 0.851 & 0.763 & 0.512 & 0.457 \\
\hline 7 & 0.670 & 0.79 & & & & \\
\hline 8 & 0.289 & 0.335 & 0.777 & 0.645 & 0.383 & 0.359 \\
\hline 9 & 0.0474 & 0.0547 & & & & \\
\hline 10 & 0.00238 & 0.00272 & 0.753 & 0.574 & 0.317 & 0.285 \\
\hline
\end{tabular}

Table 9: Comparison of our predictions for the observables $C_{0},\langle\cos \phi\rangle$ and $\langle\cos (2 \phi)\rangle$ in the NLA for $\left|\vec{k}_{J_{1}}\right|=\left|\vec{k}_{J_{2}}\right|=35 \mathrm{GeV}$ at $\sqrt{s}=14 \mathrm{TeV}$ with those of Ref. [10]. 


\begin{tabular}{|r|c|c|c|c|c|}
\hline$Y$ & $C_{0}^{(\mathrm{NLA})}$ & $\langle\cos \phi\rangle^{\mathrm{NLA}}$ & $\langle\cos (2 \phi)\rangle^{\mathrm{NLA}}$ & $Y_{0}$ & $n_{R}$ \\
\hline 6 & 0.651 & 0.845 & 0.510 & 0 & 1 \\
7 & 0.650 & & & 0 & 1 \\
8 & 0.228 & 0.967 & 0.472 & 0 & 1 \\
9 & 0.025 & & & 0 & 1 \\
10 & -0.000215 & - & - & 0 & 1 \\
\hline
\end{tabular}

Table 10: Values of $C_{0},\langle\cos \phi\rangle$ and $\langle\cos (2 \phi)\rangle$ in the NLA for $\left|\vec{k}_{J_{1}}\right|=\left|\vec{k}_{J_{2}}\right|=35 \mathrm{GeV}$ at $\sqrt{s}=14 \mathrm{TeV}$ for the kinematic values of the scales, $\mu_{R}^{2}=s_{0}=\left|\vec{k}_{J_{1}}\right|\left|\vec{k}_{J_{2}}\right|$ or $Y_{0}=0$ and $n_{R}=1$.

\begin{tabular}{|r|c|c|}
\hline$Y$ & $\begin{array}{c}C_{0}^{\text {(NLA) }} \\
{[\text { Ref. [11] }]}\end{array}$ & $\begin{array}{c}C_{0}^{\text {(NLA) }} \\
{[\text { here }]}\end{array}$ \\
\hline 6 & 0.172 & 0.186 \\
7 & 0.135 & 0.149 \\
8 & 0.0220 & 0.0247 \\
9 & 0.0007502 & 0.00088 \\
10 & $1.21610^{-6}$ & $5.7310^{-7}$ \\
\hline
\end{tabular}

Table 11: Comparison of our predictions for the observable $C_{0}$ for $\left|\vec{k}_{J_{1}}\right|=\left|\vec{k}_{J_{2}}\right|=35 \mathrm{GeV}$ at $\sqrt{s}=7 \mathrm{TeV}$ with those of Ref. [11]. 